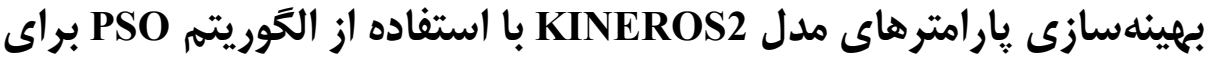

شبيهسازى رخداد سيلاب (مطالعه موردى: حوزه تمر استان تحلستان)

\author{
هادى معماريان'، محسن يوررضا بيلندى `و زينت كومه
}

\author{
ا - استاديار، دانشكده منابع طبيعى و محيط زيست دانشكاه بيرجند، (نويسنده مسوول: (hadi_memarian@birjand.ac.ir) \\ r- استاديار، دانشكده كشاورزى دانشخاه بيرجند

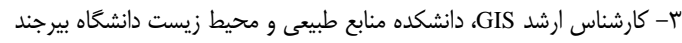

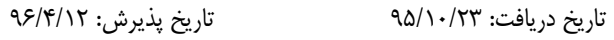

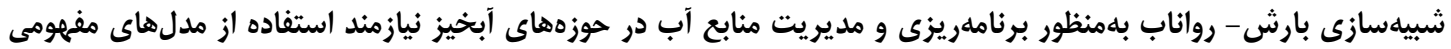

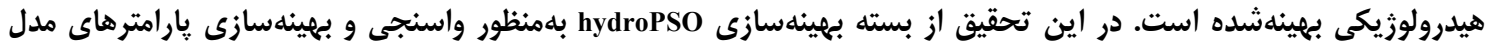

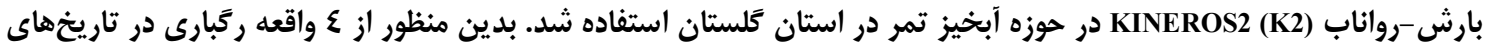

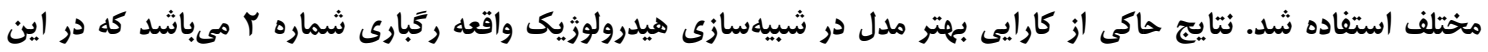

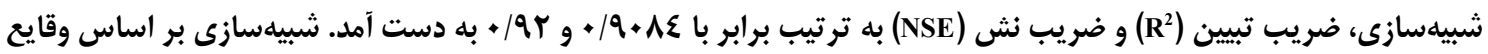

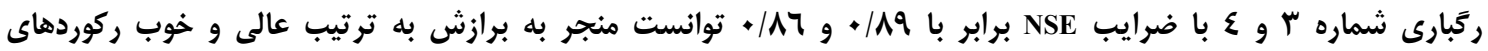

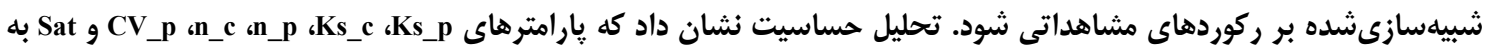

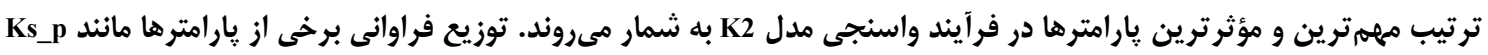

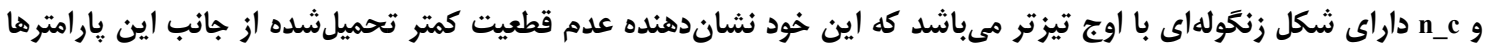

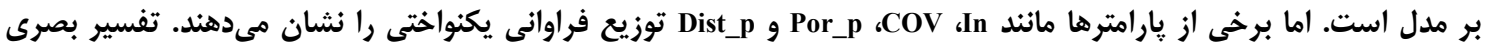

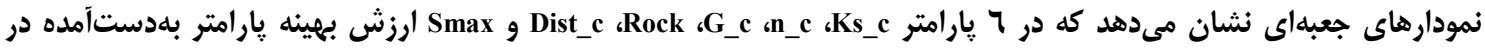

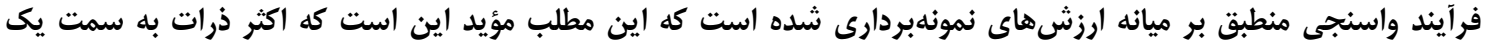

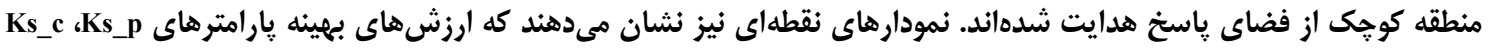

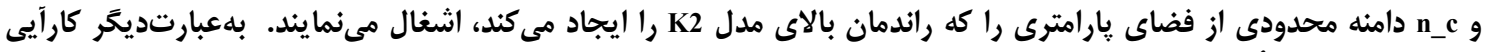

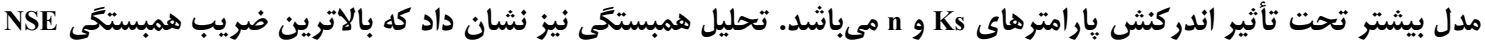

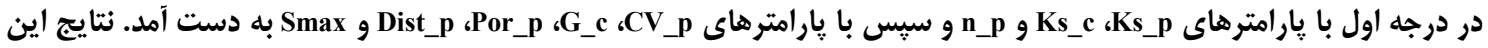

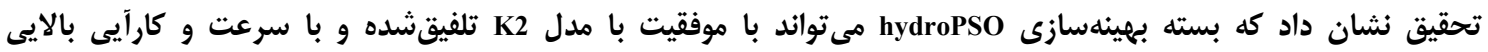

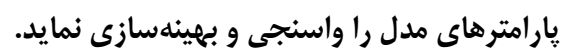

وازههاى كليدى: KINEROS2 chydroPSO، بهينهسازى انبوه ذرات، بارش -رواناب، شبيهسازى

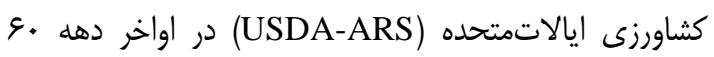

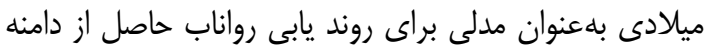

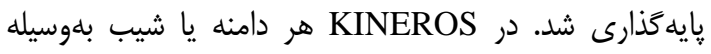

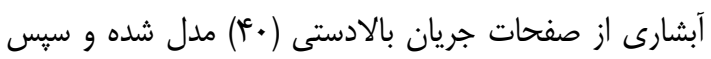

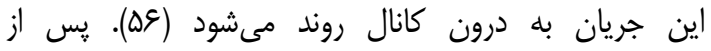

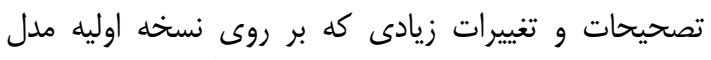

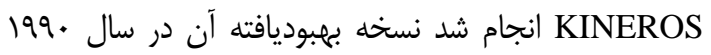
توسط وول هايزر و همكاران (DV) ارائه شد.

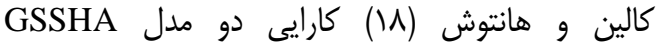
Gridded Surface/Subsurface Hydrologic (Analysis

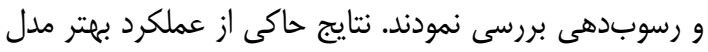

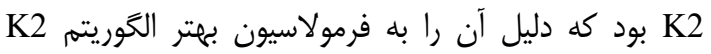

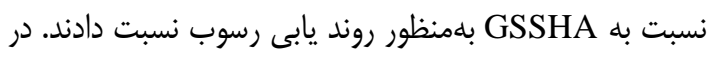

مقدمه شبيهسازى بارش-رواناب در حوزههاى آبخيز بلمنظور

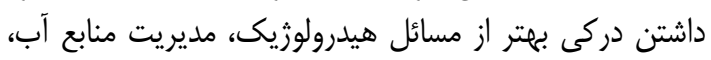

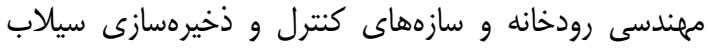

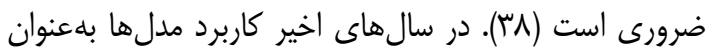

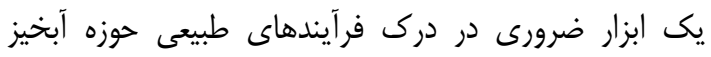

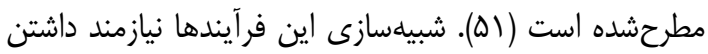

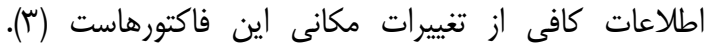

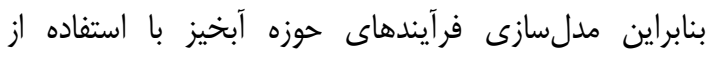

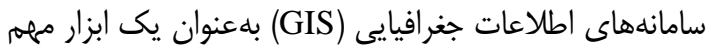

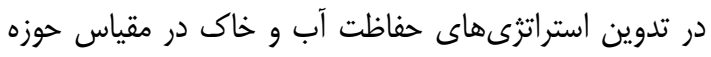

آبخيز محسوب مى تشود (TV) (TV).

مدل KINematic runoff and ) KINEROS2 يا K2 در آغاز توسط سرويس تحقيقات (EROSion 
روبرو كرده است. بهعنوانمثال مشكل وجود جندين بهينه

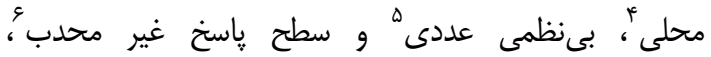

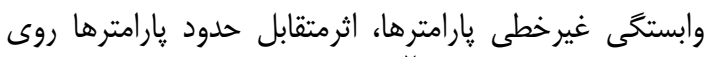

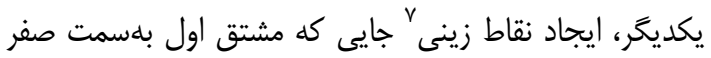

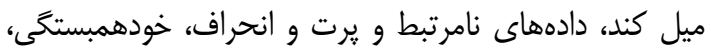

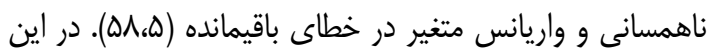

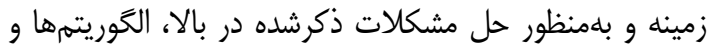

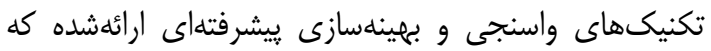

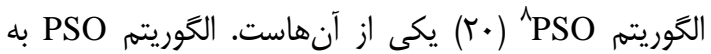
علت قابليت انعطاف، اجراى آسان و كارايى بالا در إنى ساليان

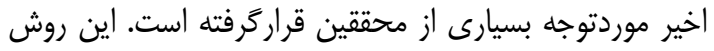

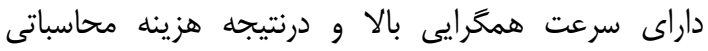

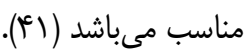
مهمترين نرمافزارى كه تاكنون توانسته است از الخوريتم

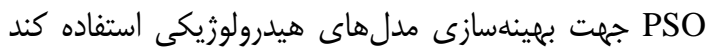
بسته نرمافزارى R است كه با كمك ابزار يك بسته مستقل است مىتواند آناليز حساسيت، واسنجى

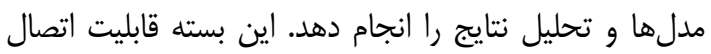

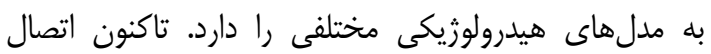

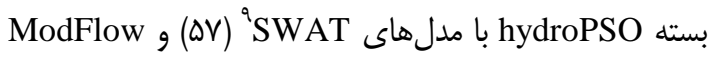
(1) انجامشده است. كاربردهاى الگوريته PSO

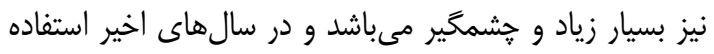

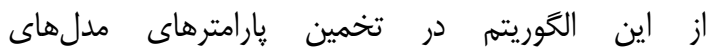

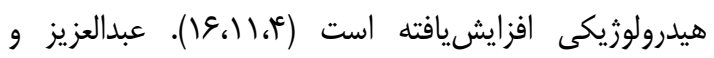

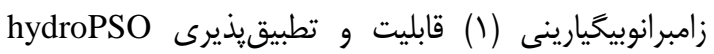

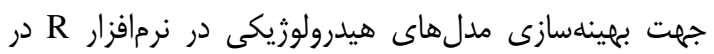
TU Bergakademie Freiberg را مورد مطالعه قراردادند. نتايج نشان داد كه علاوه

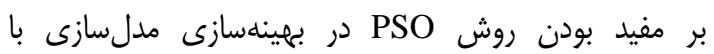
MODFLOW كاهش تعداد تكرارهاى موردنياز براى رسيدن به مقدار بهينه

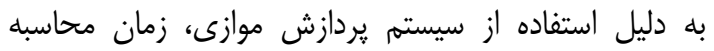

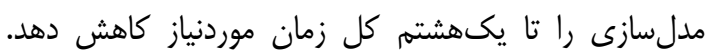

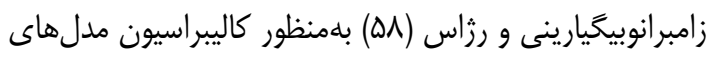
هيدرولوزيكى از بسته hydroPSO بهعنوان يك بـ بسته مجزا

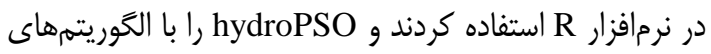
(SCE_UA, DE, DREAM, SPSO-2011, استاندارد با استفاده از يكسرى توابع خاص مقايسه and GML)

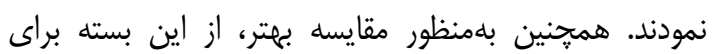

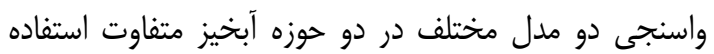

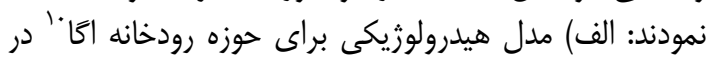

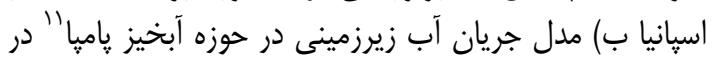

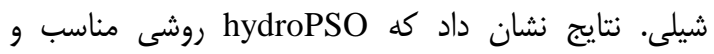

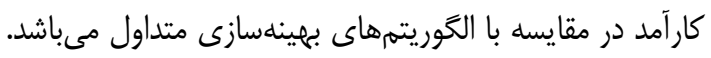

مطالعه ديخرى اسميت و همكاران (^أ) توانايى مدل KINEROS2

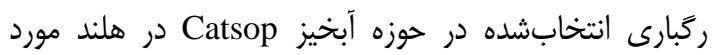

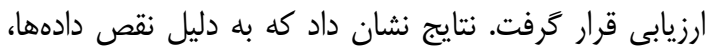

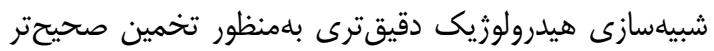

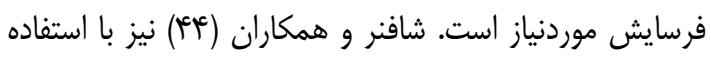

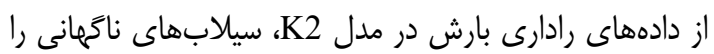

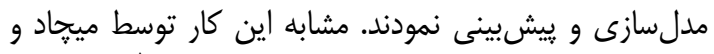

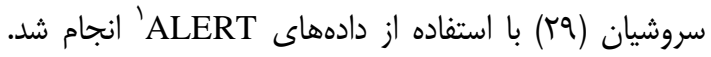
ييشبينى خطر سيلاب تحت تأثير سناريوى تغيير كاربرى اراضى نيز با استفاده از مدل

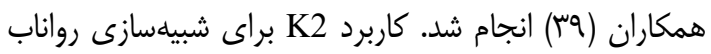

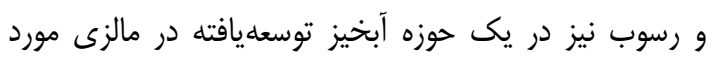

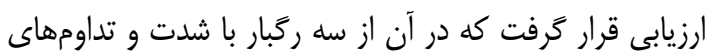

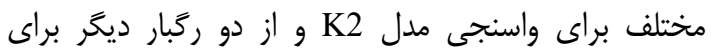

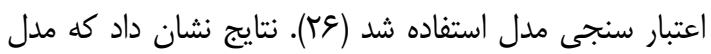

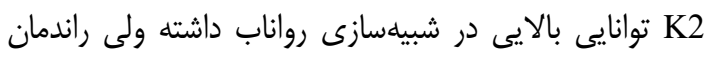

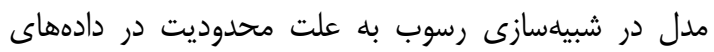

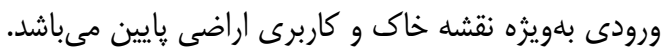

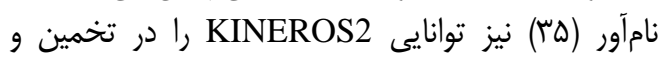
شبيهسازى رواناب در حوزه آبخيز كامه در ايران بان استفاده

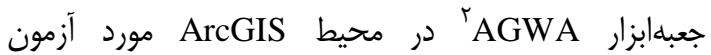

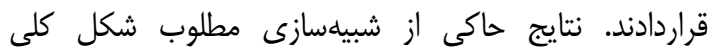

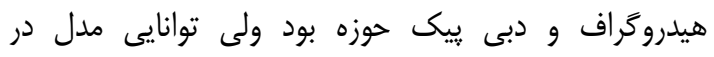
شبيهسازى رسوب به علت فقدان دادههاى مشاهداتى مورد

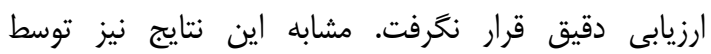

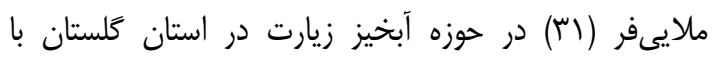

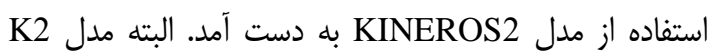
در مقياس كوجى نيز در تحقيقات مختلفى مورداستفاده،

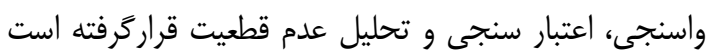

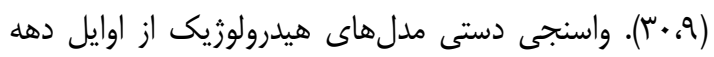

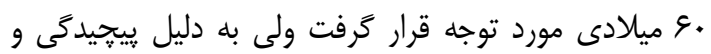

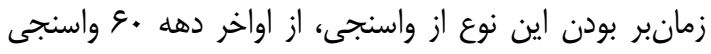
خودكار مدلهاى هيدرولوزيك مطرح شد و توسعه ييدا كرد. هر نوع واسنجى خودكار نياز به تعريف تابع هدف، الخوريتم

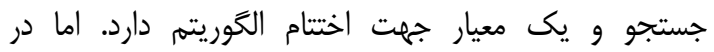

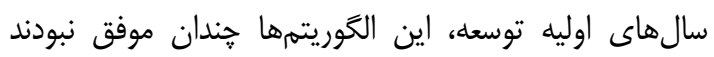
(If)

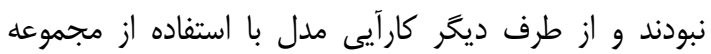

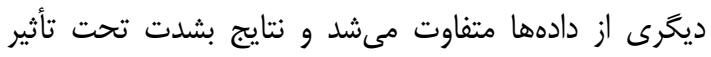

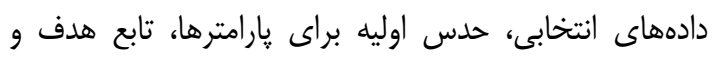

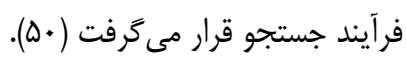

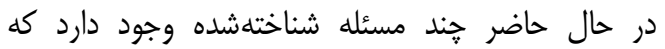

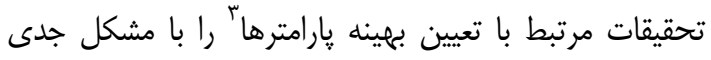

1- Automated Local Evaluation in Real-Time 3- Optimum parameter set

6- Non-convex response surface

9- Soil and Water Assessment Tools
2- Automated Geospatial Watershed Assessment

4- Local optima 5- Numerical granularity

7- Saddle points 8- Particle Swarm Optimization

10- Ega River Basin
11- Pampa 


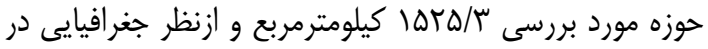
محدوده 'بr

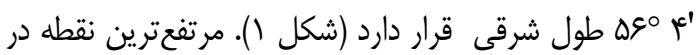

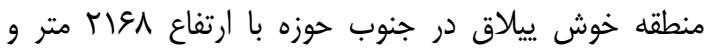

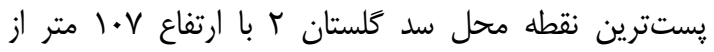

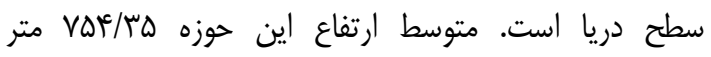
مىباشد. در حوزه آبخيز تمر تعداد محدودئ إنفاع ايستخاه

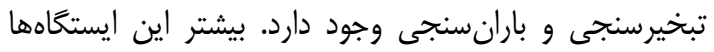

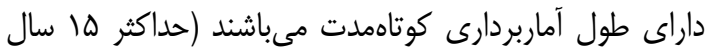

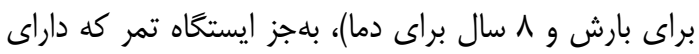

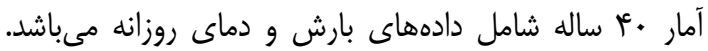

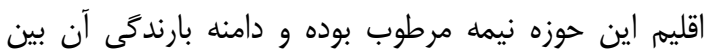

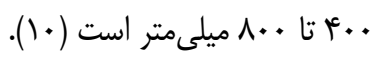

همجنين اين بسته نرمافزارى مقياسيذير مياشد؛ يعنى كارايى مدل با بالا رفتن ابعاد مسئله حفظ مىشود و ودر در مسائل مختلف قابل انطباق مى باشاشد.

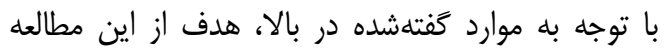

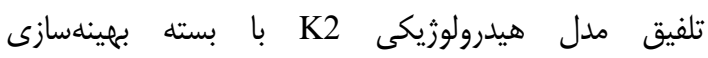
إخيز hydroPSO

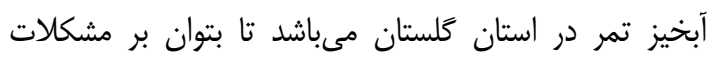

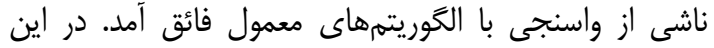

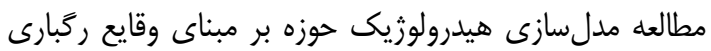

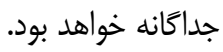

\section{مواد و روشها مالماني} منطقه مطالعاتى حوزه آبخيز تمر يكى از زيرحوزههاى كركان روان رود است كه

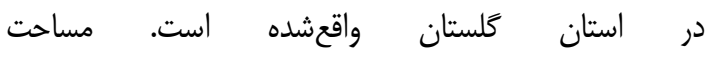

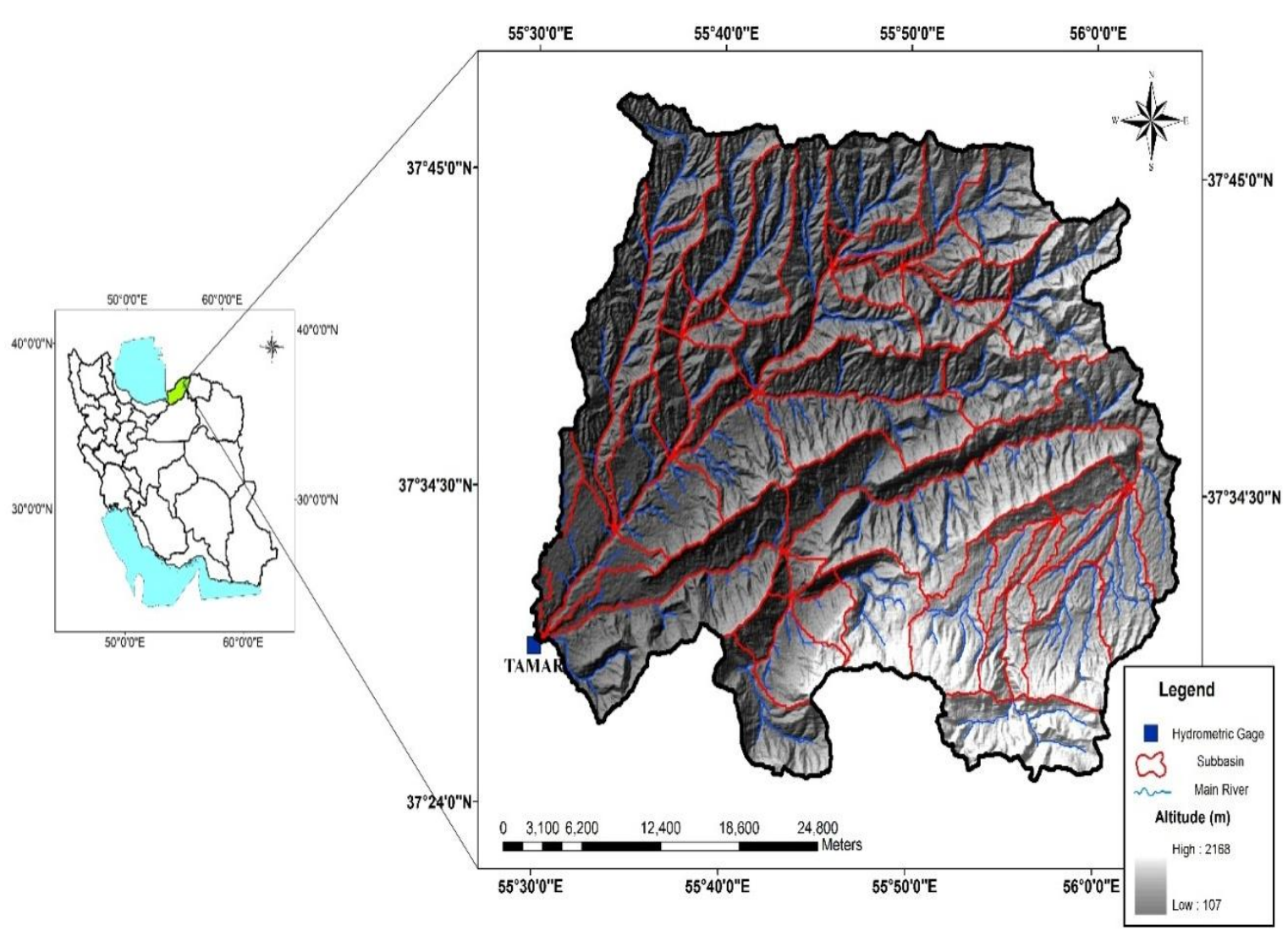

شكل (- موقعيت حوزه آبخيز مطالعاتى تمر در استان كلستان و كشور

Figure 1. Geographic location of study area Tamar Watershed, Golestan, Iran 
ارث تهيه شد (شكل ؟). در تهيه نقشه سرىهاى خاك از

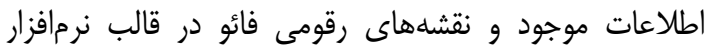
(Yץ) Harmonized World Soil Database (HWSD) كمى گرفته شد. مدل رقومى ارتفاع نيز از مجموعه دادههاى

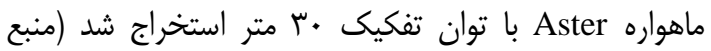
تمييه داده: (http://gdex.cr.usgs.gov/gdex

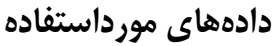

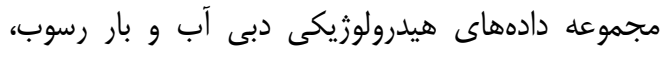

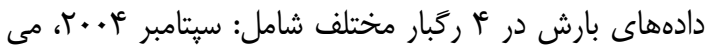

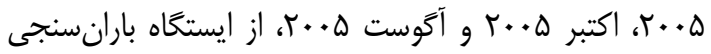

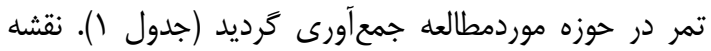

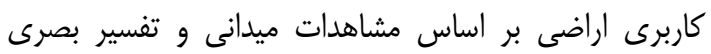
تصاوير ماهوارهاى SPOT بكار كَرفتهشده در نرمافزار كوّل

Table 1. Properties of selected storm events

جدول 1- ويزگى هاى ركبارهاى انتخابشده جهت شبيهسازى هيدرولوزيك

\begin{tabular}{|c|c|c|c|c|c|}
\hline $\mathrm{I}_{60 \_} \max (\mathrm{mm} / \mathrm{h})$ & حجم بارش (MCM) & عمق بارش (mm) & زمان تداوم (h) & تاريخ وقوع & شماره رَّبار \\
\hline $\mid r / r$ & $V g / V$ & $\Delta \cdot / T \Lambda$ & IV & 19 SEP 2004 & 1 \\
\hline$N / I F$ & $\Lambda \mathrm{V} / \mathrm{s}$ & $\Delta V / \& Y$ & ع & 06 MAY 2005 & r \\
\hline$V / q V$ & $g r / \Lambda$ & Fi/IV & 15 & 08 OCT 2005 & r \\
\hline १/१ & $9 . / 9$ & $\Delta Q / \bar{Q}$ & $r$. & 09 AUG 2005 & f \\
\hline
\end{tabular}
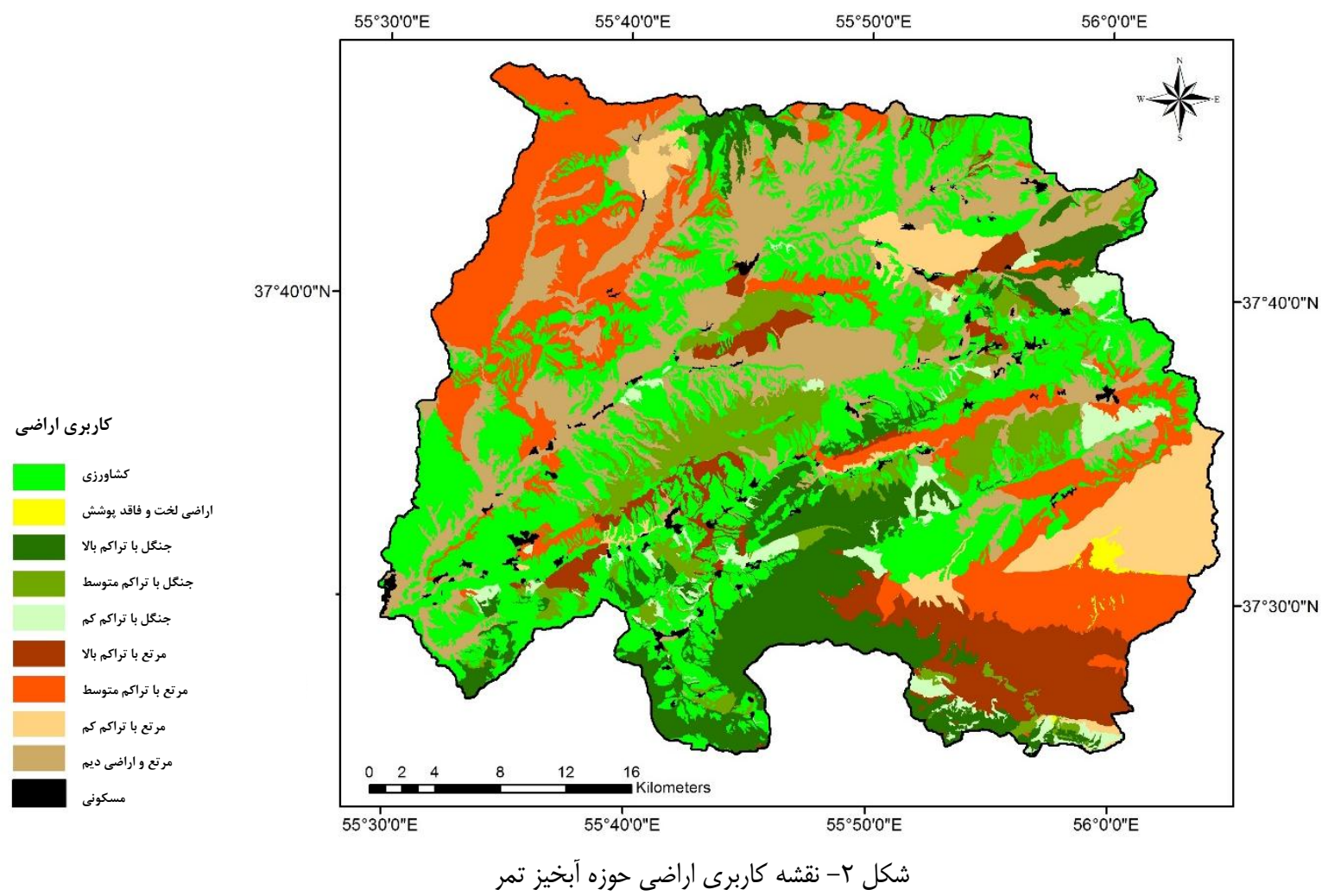

Figure 2. Land use map of Tamar watershed

حاصله نيز بر اساس معادله هورتن محاسبه مى مباشد. براساس

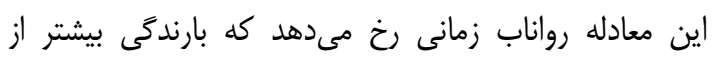

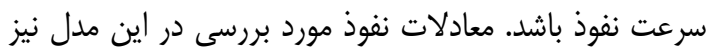

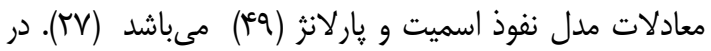

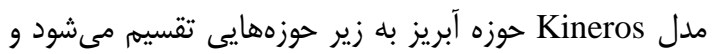

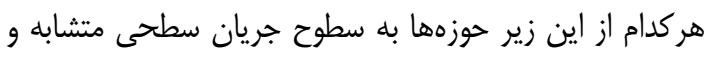

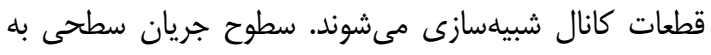

توصيف مدل KINEROS Kineros

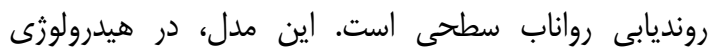

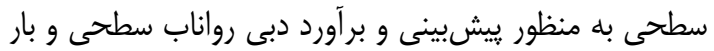

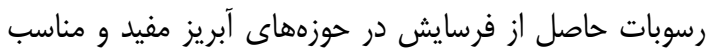

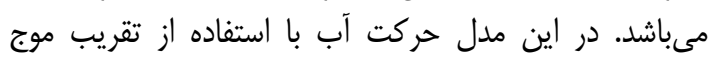

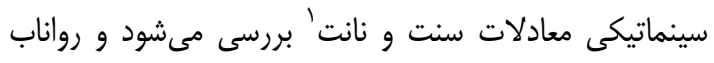




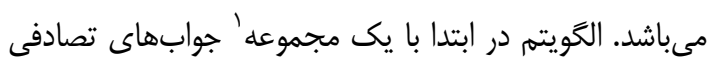

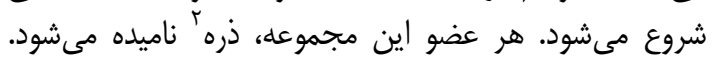

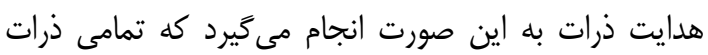
بهترين موقعيتى را كه در طى فرآيند جستجو كسب كردي كرداند

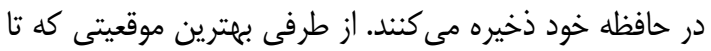

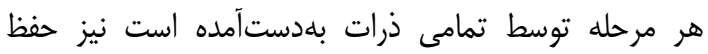

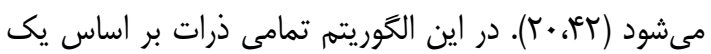

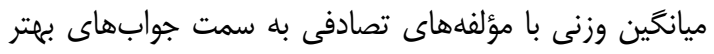

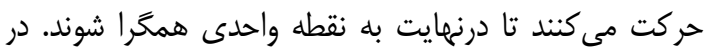

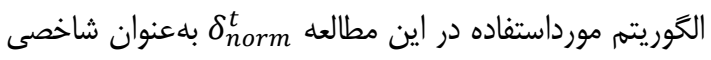

براى تعيين ميزان همكر ايى الكوريتم استفاده مى شود (().

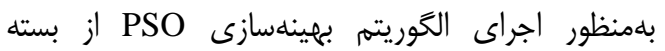
hydroPSO hydroPSO

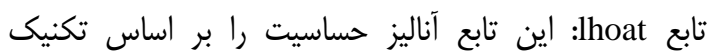

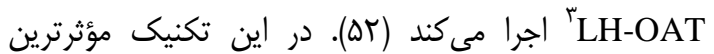

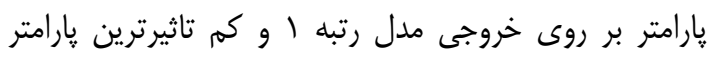

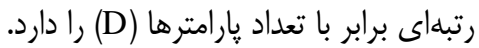

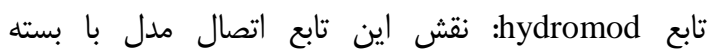
hydroPSO

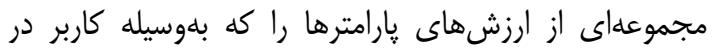

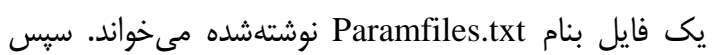
تابع hydromod فايل اجر ايى مدل را فراخوانى كرده تا بتواند

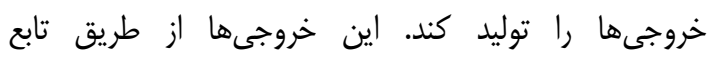
out.FUN شبيهسازىشده با خروجىهاى واقعى (مشاهداتى) از طريق

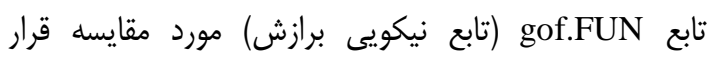

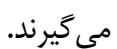

Nash-Sutcliffe مر اين مطالعه تابع هدف مورد استفاده Efficiency (NSE) تابع hydroPSO كه موتور اصلى واسنجى مدل هيدرولوزيك

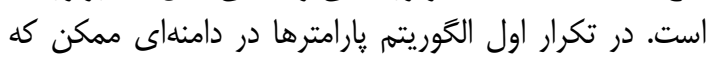
توسط كاربر در فايل ParamRanges.txt تعريفشده است

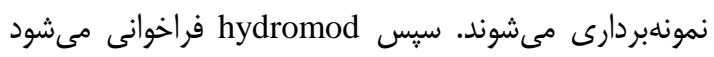

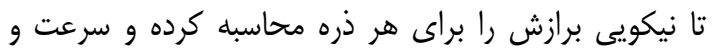

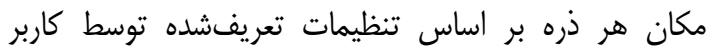

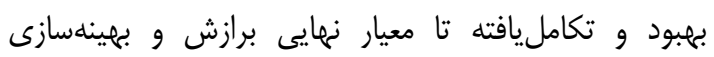

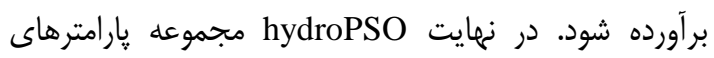

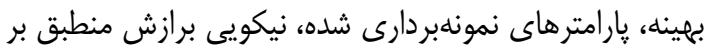

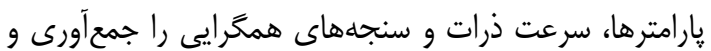

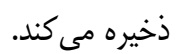

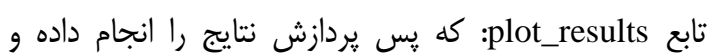

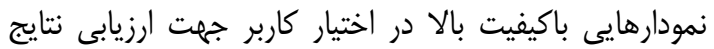
واسنجى قرار مىدهد.

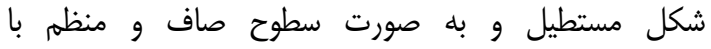

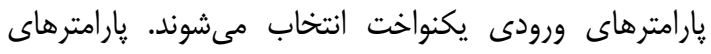

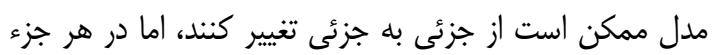

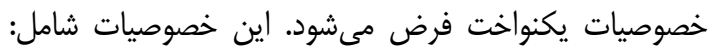

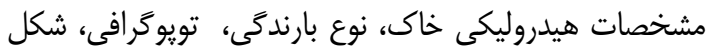

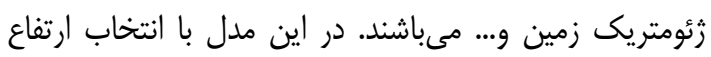

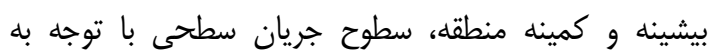

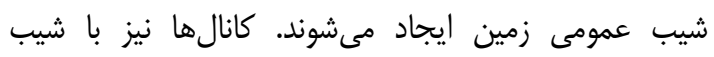

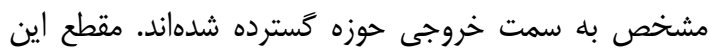

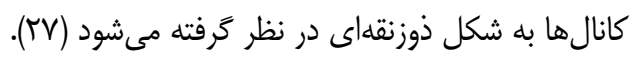
Kineros Kineros2

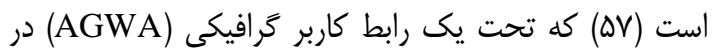

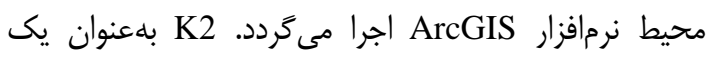

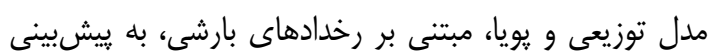

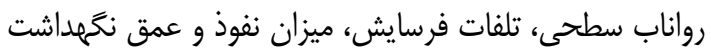

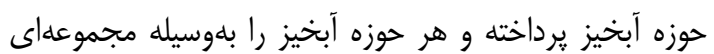

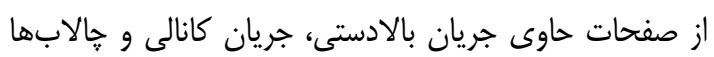

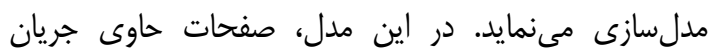

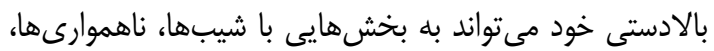

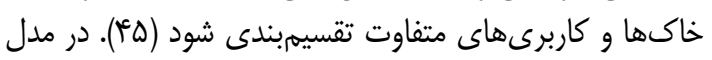

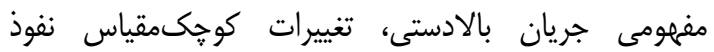

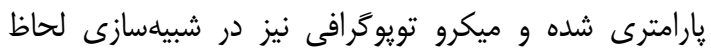

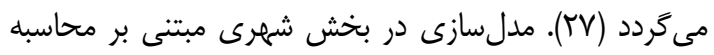

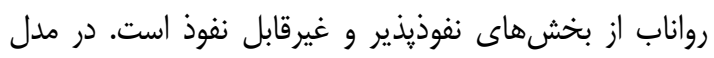

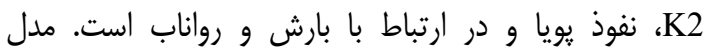

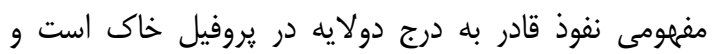

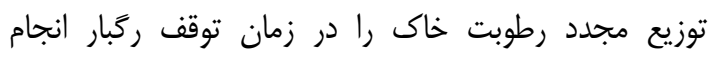

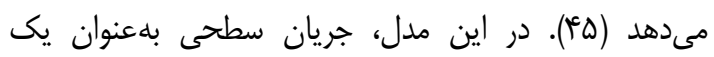

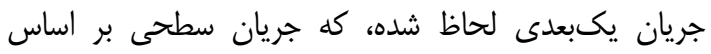

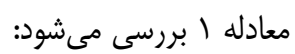
$\frac{\partial \mathrm{h}}{\partial \mathrm{t}}+\frac{\partial \mathrm{Q}}{\partial \mathrm{x}}=\mathrm{q}(\mathrm{x}, \mathrm{t})$ ) رابطه (1)

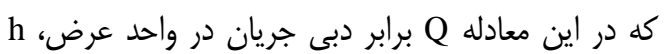

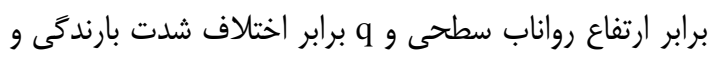

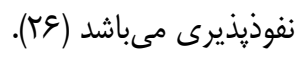

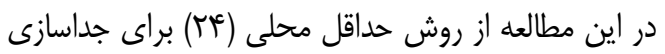

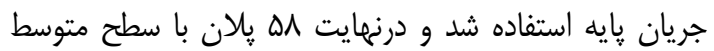

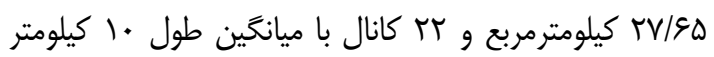
در محيط AGWA جهت مدلسازى در سطح حوان حوزه آبخيز تمر جدا شد.

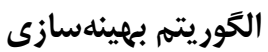

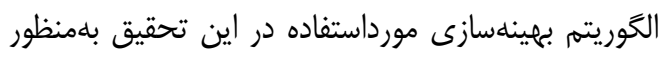

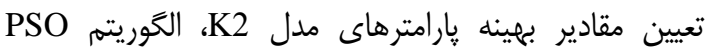




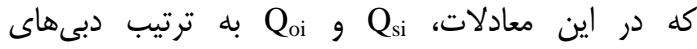

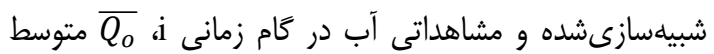

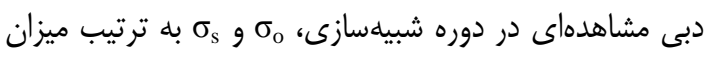

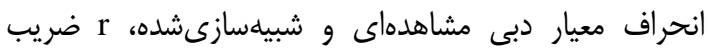

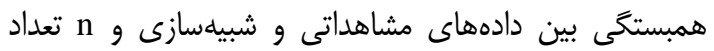
مشاهدات در دوره شبيهسازى است. ارزش ايده دآل دآل براى

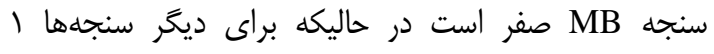

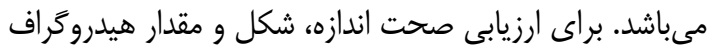

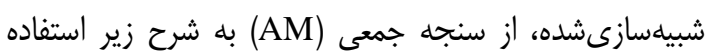

شد:

$A M=\frac{\mathrm{r}_{\mathrm{mod}+\mathrm{NS}+(1+|\mathrm{MB}|)}}{3}$

(ه) (ه) (ه):

ميزان ايده آل اين سنجه نيز ا مىباشد كه نشاندهنده

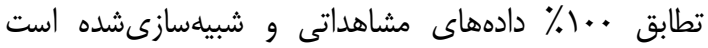

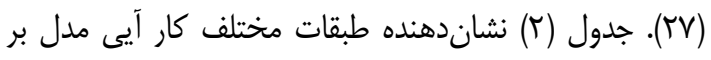
اساس سنجه AM - (
تابع verification: بلمنظور اعتبارسنجى مجموعه يارامترهاى تعريفشده توسط كاربر با استفاده از محاسبه نيكويى برازش.

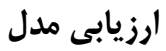

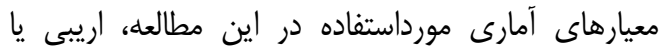

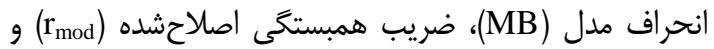

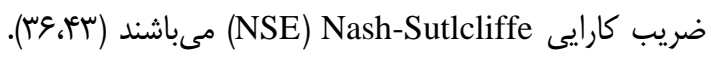
معادلات زير توصيف كننده اين معيارها بلهورت عددى است:

$$
M B=\left[\sum_{i=1}^{n}\left(Q_{s i}-Q_{o i}\right) / \sum_{i=1}^{n} Q_{o i}\right] \quad(Y) \text { رابطه }
$$

$r_{\text {mod }}=\left[\frac{\left(\min \left(\sigma_{o}, \sigma_{S}\right)\right.}{\max \left(\sigma_{o}, \sigma_{S}\right)} * r\right]$

رابطه (1)

Table 2. Model performance categories based on the AM measure

جدول r- طبقات مختلف كار آيى مدل بر اساس سنجه AM ( Tأ، TV)

\begin{tabular}{|c|c|}
\hline نيكويى برازش & Aggregated Measure (AM) \\
\hline عالى & $>\cdot / \Lambda \Delta$ \\
\hline خيلى خوب & $\cdot / V \cdot-\cdot / \Lambda \Delta$ \\
\hline خوب ل & $\cdot / \Delta D_{-} \cdot / \mathrm{V}$ \\
\hline ضعيف & $\cdot / \mathbb{C} \cdot-\cdot / \Delta \Delta$ \\
\hline خيلى ضعيف & $<\cdot / \kappa$. \\
\hline
\end{tabular}

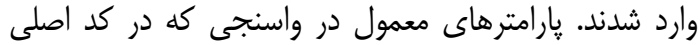

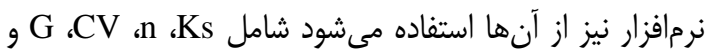

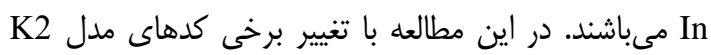

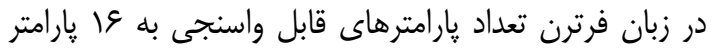

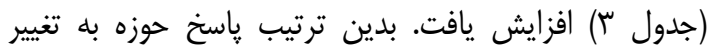

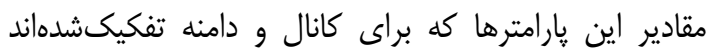

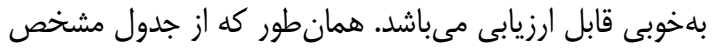

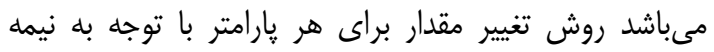

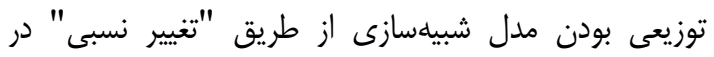

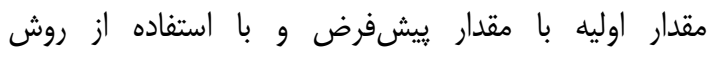
ضرب كننده يا Multiplier انجام شد.
يار امترهاى مدل در فرآيند بهينهسازى

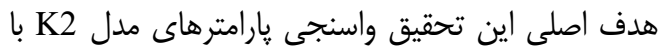

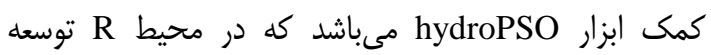
دادهشده و تاكنون نيز موارد معدودى از استفاده از اين ابزار در مر مرايط

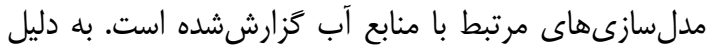

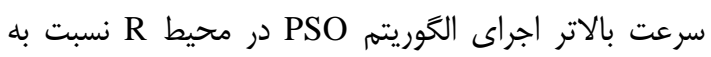
محيط نرمافزارى MATLAB و همجنين دسترسى و استفاده

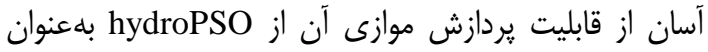

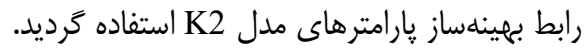

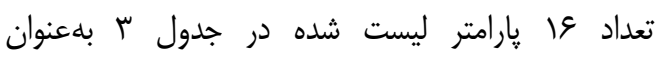

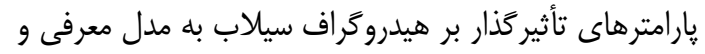


جدول ץ- پارامترهاى مدل K2 جهت ورود در فرآيند بهينهسازى توسط hydroPSO Table 3. K2 optimization parameters used by hydroPSO

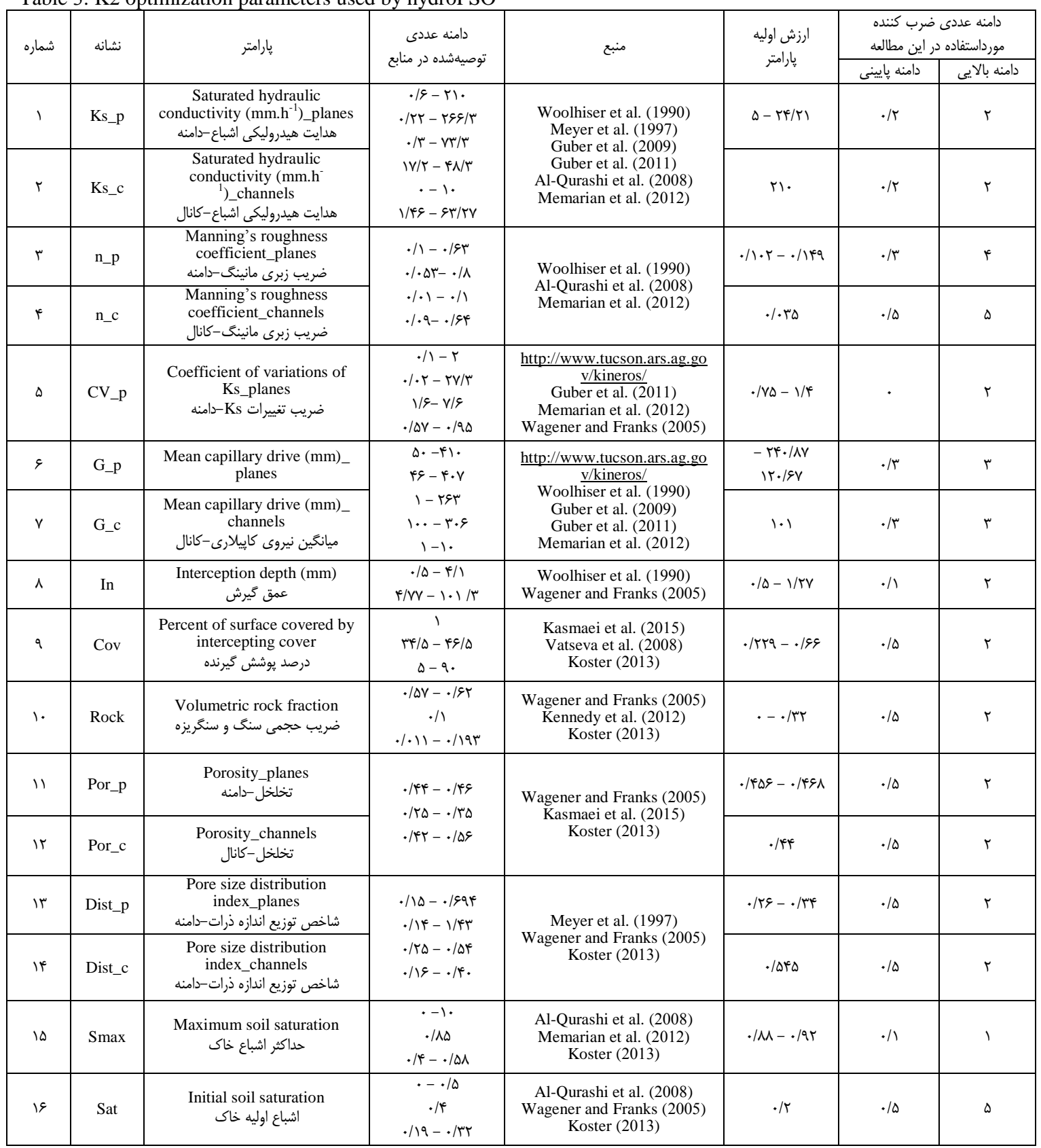


ضعيفترين نيكويى برازش در ميان وقايع شبيهسازى شده

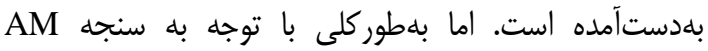

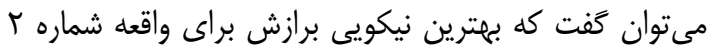

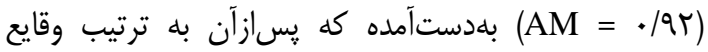

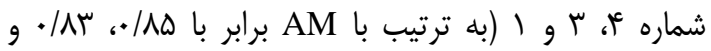

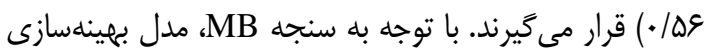

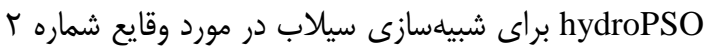

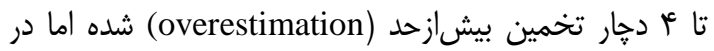

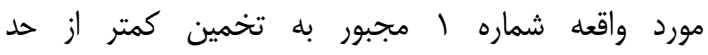
سيلاب شده است. برخى انحرافها نيز إنديز (underestimation)

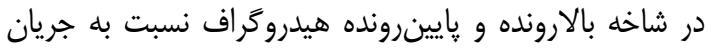

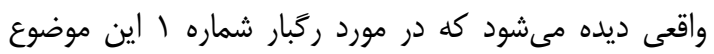

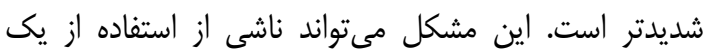

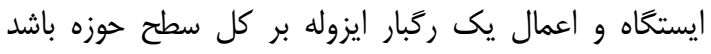

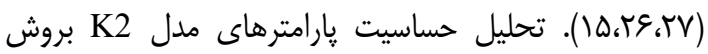

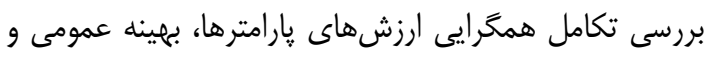

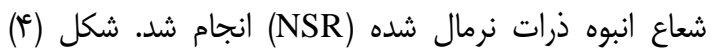

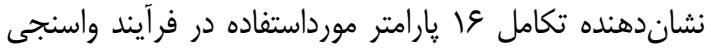

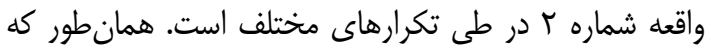

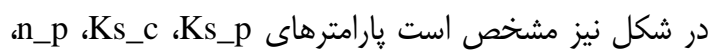
و CV CV_p ،n_c

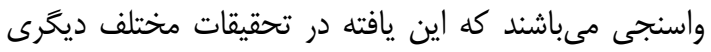

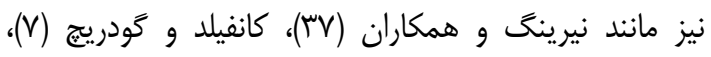

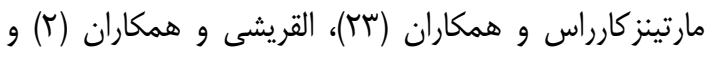

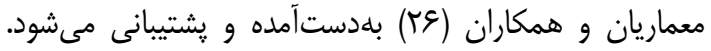

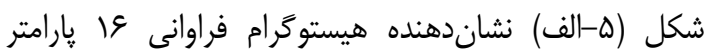

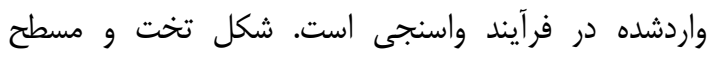

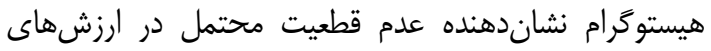

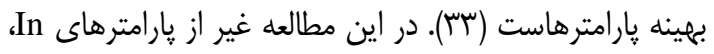

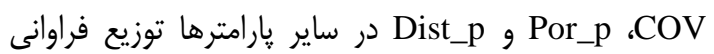

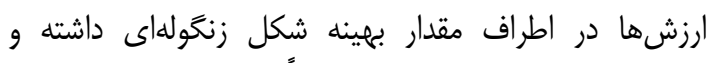

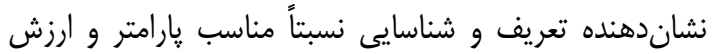

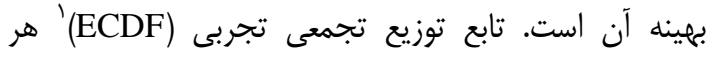

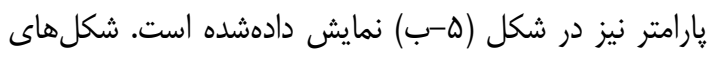

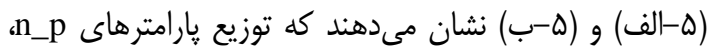
Sat و Smax ،Dist_c ،Por_c ،Rock ،G_c ،CV_p ،n_c

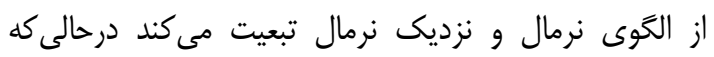
بارامترهاى Ks_p و

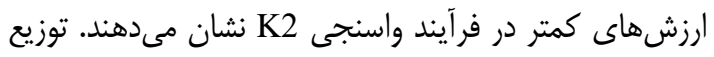

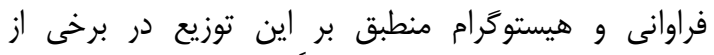

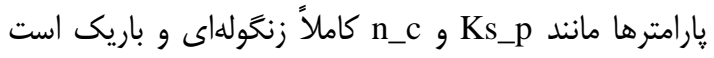

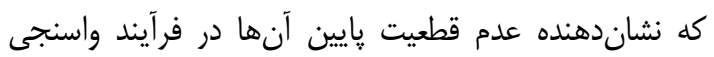

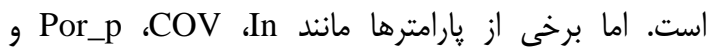
توزيع يكنواختى را در فرآيند نمونهبردارى و واسنجى Dist_p
نتايج و بحث

يكى از معمولترين روشهاى ارزيابى كارايى يكى مدل

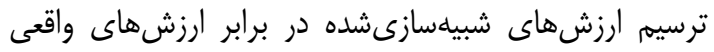

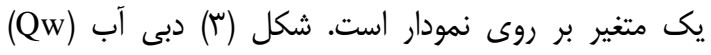

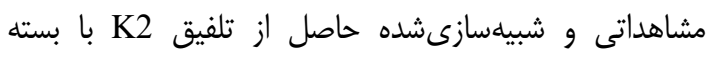
hydroPSO

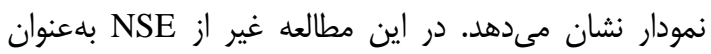

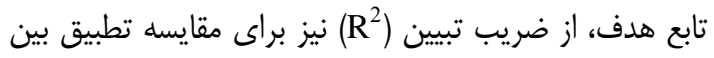

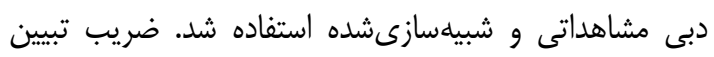

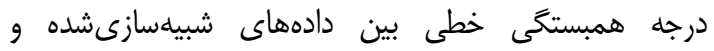

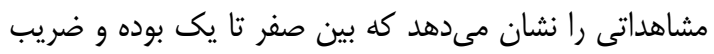

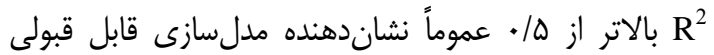

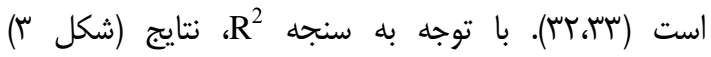
نشاندهنده تطبيق بهتر دبى آب شبيهسازىشده با دبى آبـ آب مشاهداتى براى واقعه شماره بَ مى باشد.

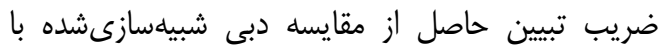

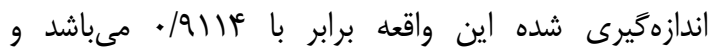

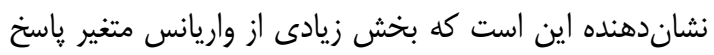

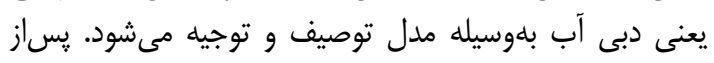

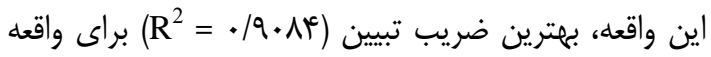

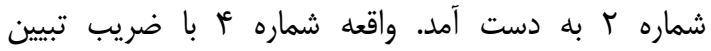

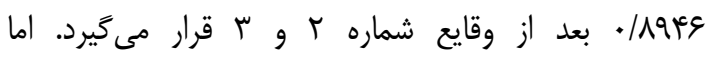

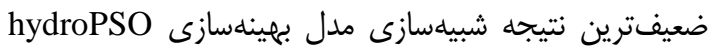

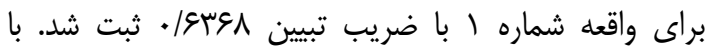

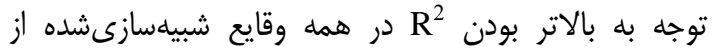

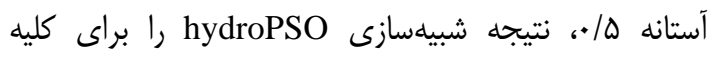

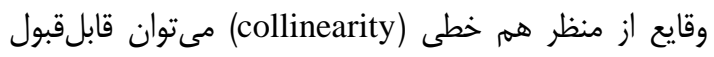

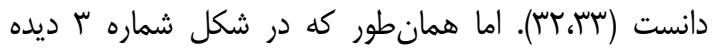

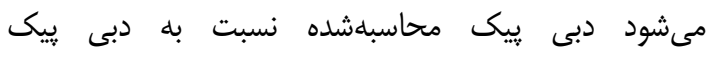

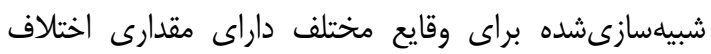

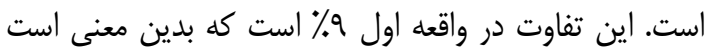

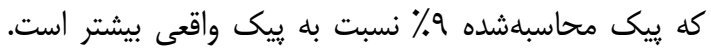

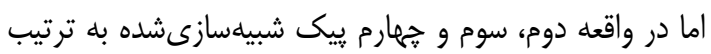

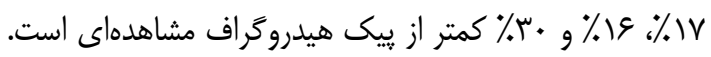

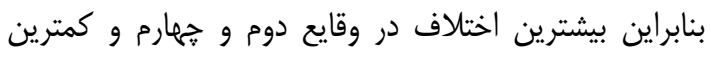

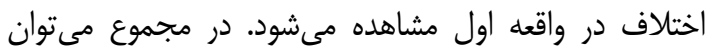

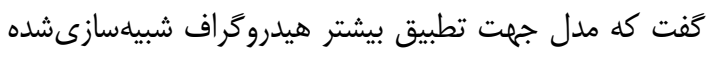
با هيدروكراف مشاهدهاى تمايل بيشترى به تخمين كمتر دبى بـى

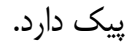

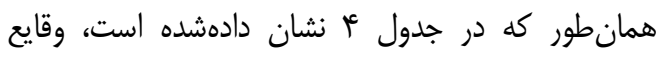

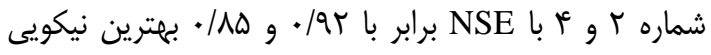

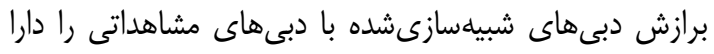

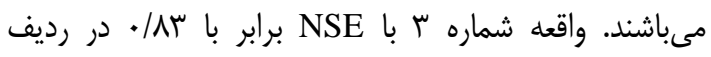

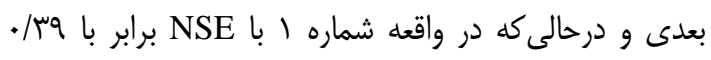




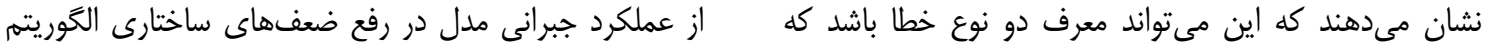

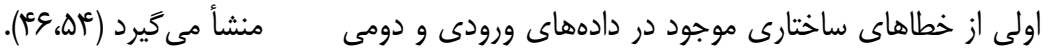
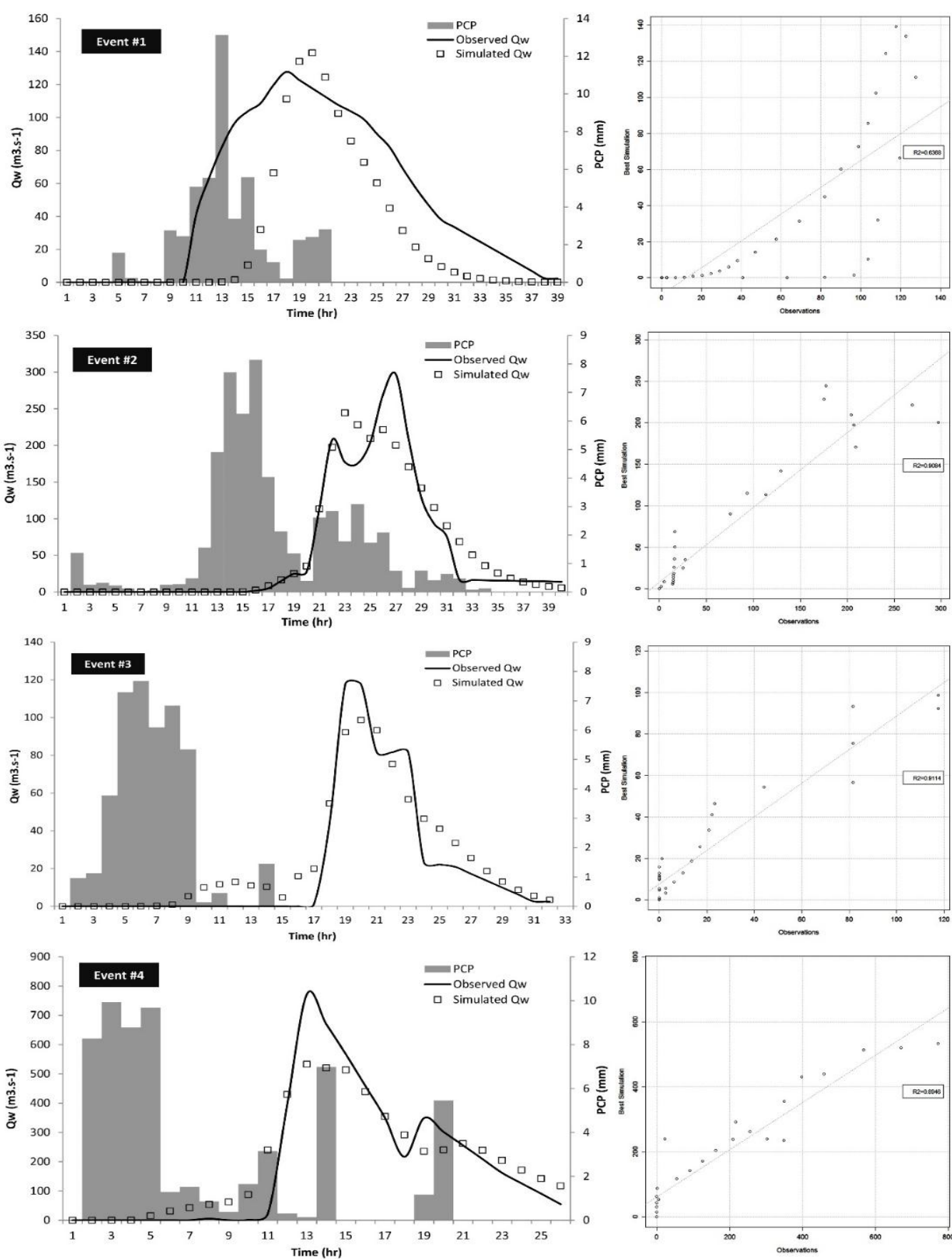

شكل ץ- دبى شبيهسازى شده (Simulated Qw) در برابر دبى مشاهدام (Observed Qw) همراه با هيتوكر اف بارش (PCP) بر اساس وقايع مختلف ركبارى

Figure 3. Observed vs. simulated water discharge with the hyetographs of selected storm events 
جدول عا- سنجهای ارزيابى مدل براى شبيلسازى مبتنى بر وقايع بارشى ا تا ع Table 4. Fitting metrics of selected storm events (the events \#1- \#4) for runoff modeling

\begin{tabular}{|c|c|c|c|c|}
\hline واقعه Fا & واقعه r & واقعه r & واقعه 1 & سنجه ارزيابى مدل \\
\hline$\cdot 1 \cdot \Delta$ & $\cdot / r$ & $\cdot 1 \cdot)^{c}$ & $-\cdot / A q$ & MB \\
\hline$\cdot / V r$ & $\cdot|\Lambda|$ & $\cdot / 9$. & $\cdot / \mathrm{V}^{\mathrm{c}}$ & $r_{\text {mod }}$ \\
\hline$\cdot / \Lambda \Delta$ & •/^ץ &.$/ 94$ & $\cdot / \Delta \varphi$ & $\mathrm{AM}$ \\
\hline عالى & خيلى خوب & عالى & خوب & نيكويى برازش \\
\hline
\end{tabular}
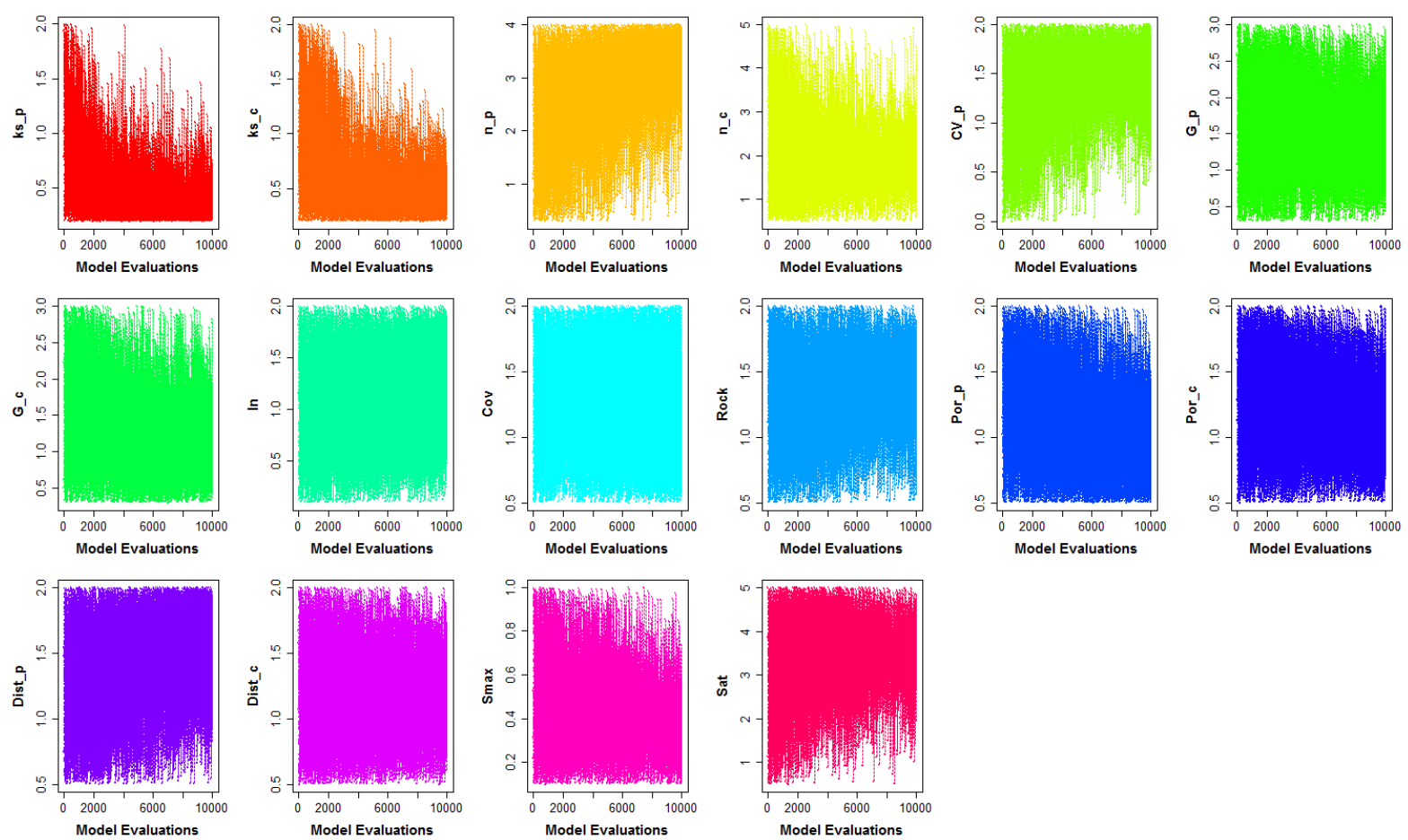

شكل ع- ارزش يارامترها در هر اجراى مدل در طى فرآيند واسنجى بر اساس شبيهسازى هيدرولوزيك مبتنى بر واقعه شماره r

Figure 4. Parameters values per run in model calibration based on the event \#2

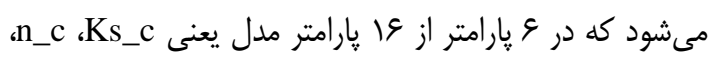
و مmax Dist_c ،Rock ،G_c ميانه ارزشهاى نمونه

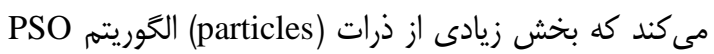

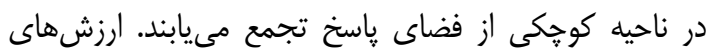

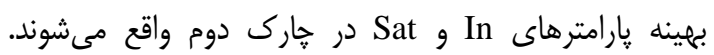
شكل (צ-ب) نشان مىدهد كه ارزشهاى بهينه پارامترهاى و و Sat CV_p ،n_c ،Ks_c ،Ks_p شدهاند اما در مورد ساير يارامترها مقدارى عدم قطعيت وجود

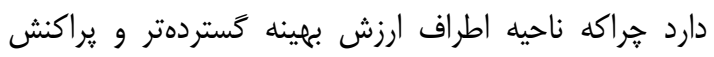
ارزشهاى نمونهگيرى شده بيشتر است.
نمودارهاى جعبهاى در شكل (و-الف)، توزيع آمارى ارزشهاى نمونهبردارى شده را به روش ديخرى برى براى واقعه

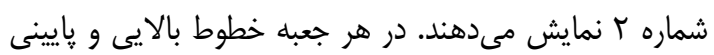

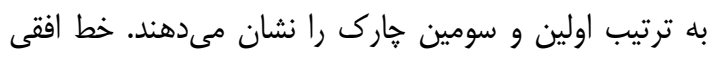

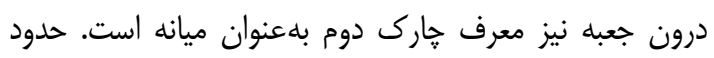

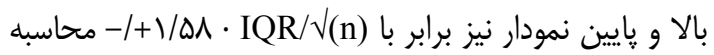

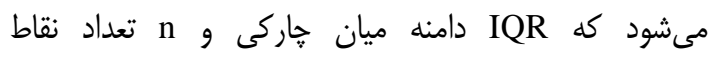

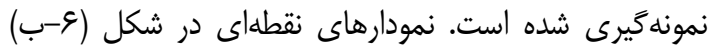
نشاندهنده ارزش يارامتر در برابر نيكويى برازش ندوان (NSE)

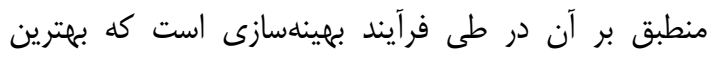

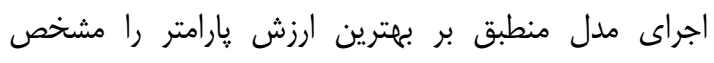

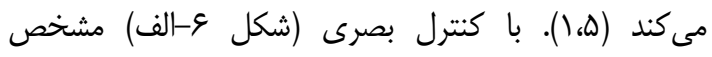



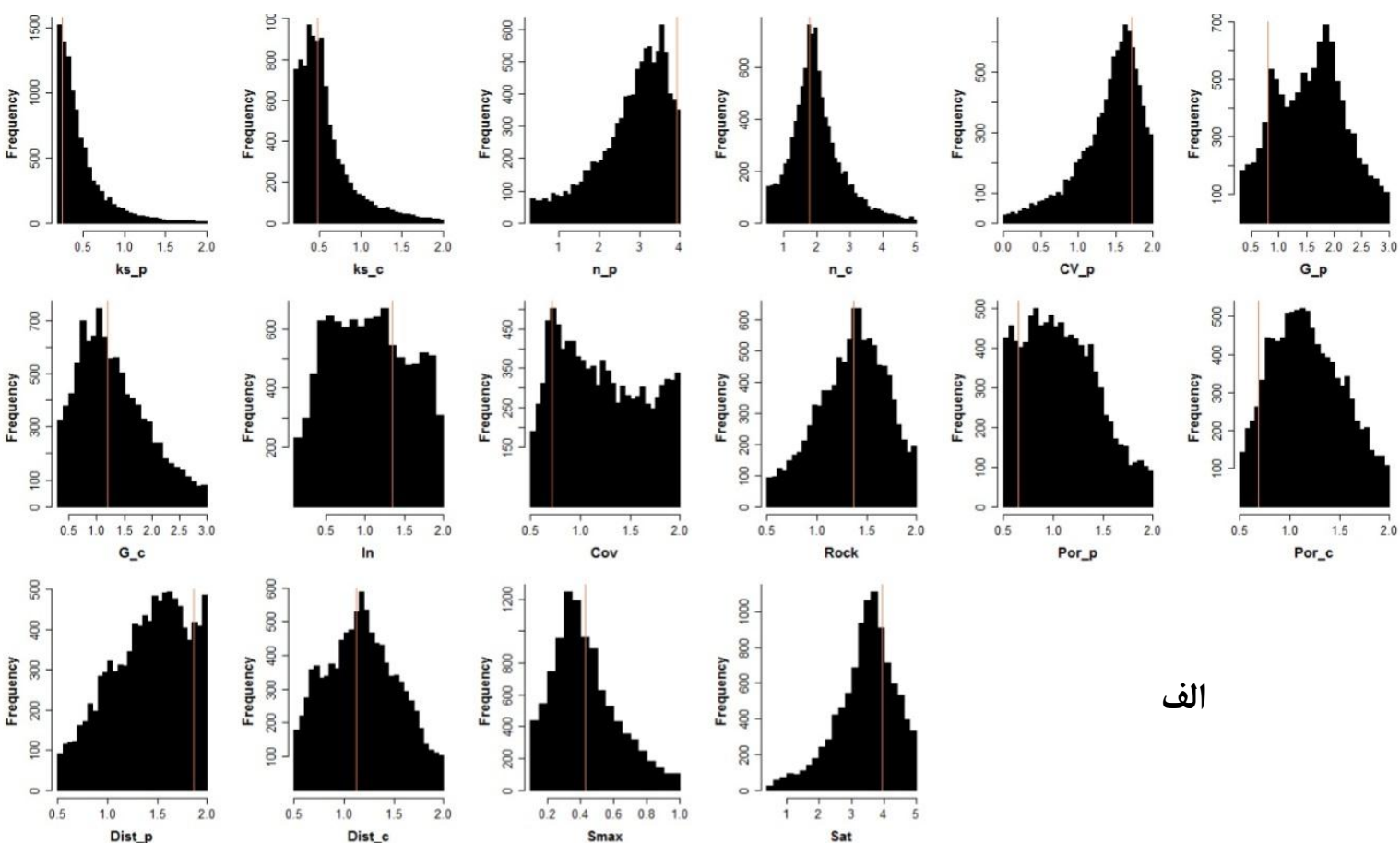

الف
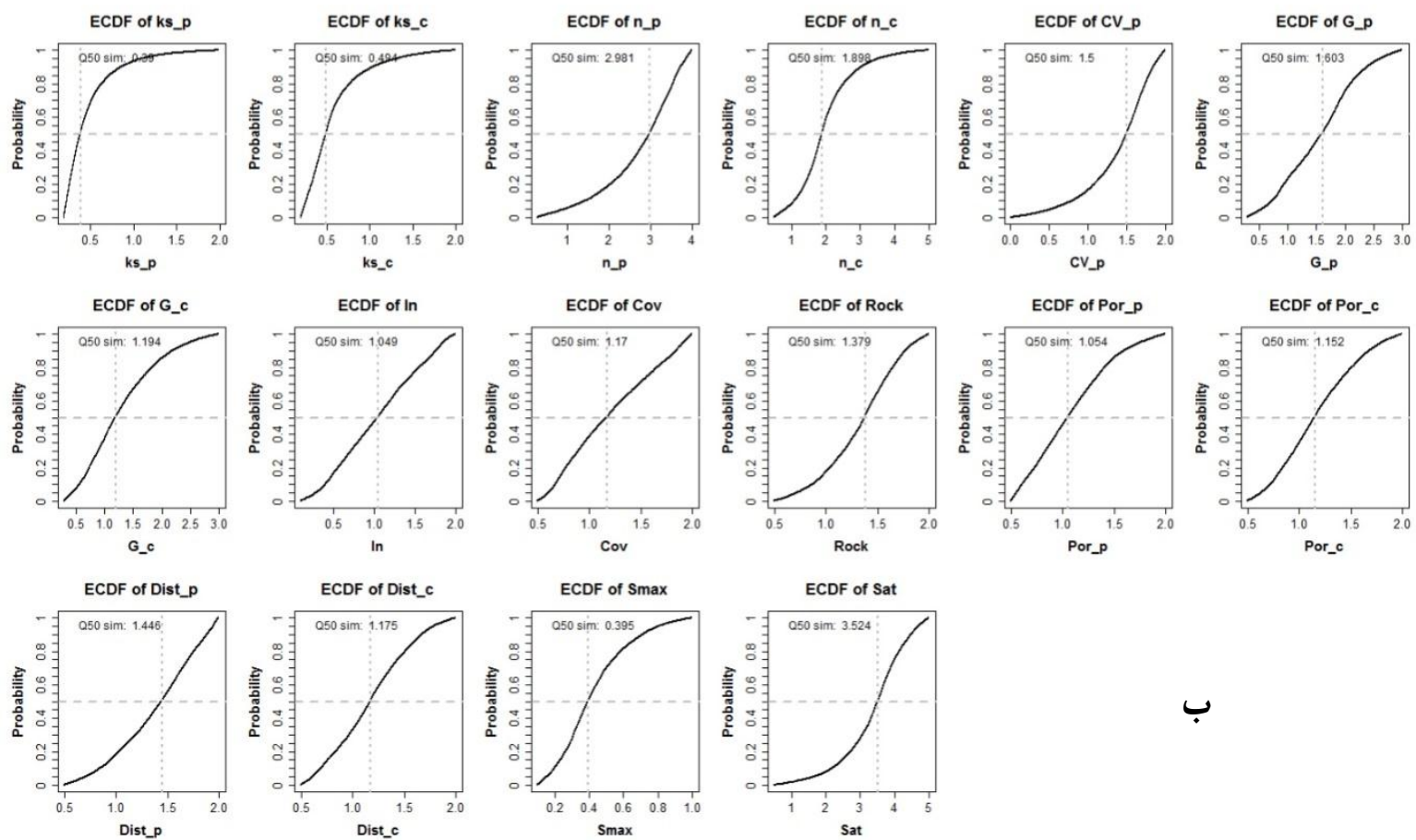

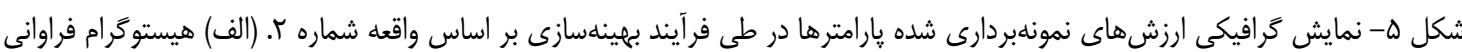

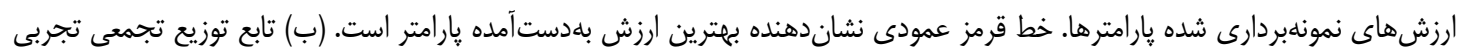

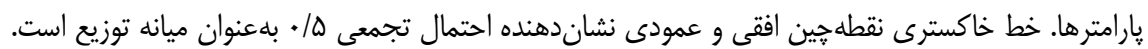

Figure 5. Graphical summary of parameter values sampled during the optimization. (a) Histograms showing the frequencies of the parameter values. Vertical red line indicates the optimum value found for each parameter. (b) ECDFs of parameter values. Horizontal gray dotted lines represent a cumulative probability equal to 0.5 (median of the distribution). Vertical gray dotted lines represent a cumulative probability of 0.5 . 
دادهشه است. بخش بالاى قطر ماتريس نشاندهنده ضريب

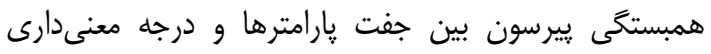

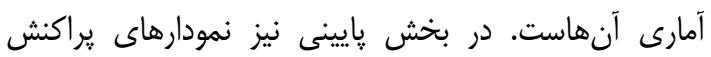

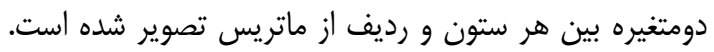

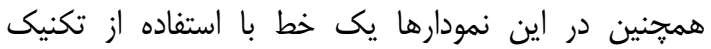

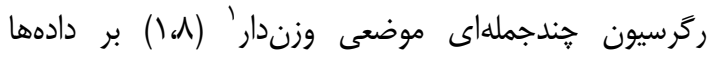
برازش يافته است. هيستوكرام هر فاكتور كه طنى فرآ فرآيند

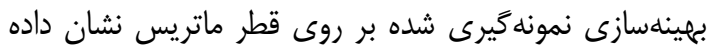

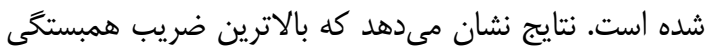

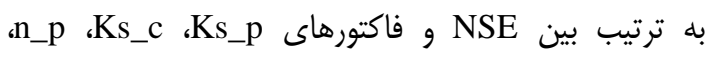
هor و و Dist_p ،Por_p ،G_c ،CV_p

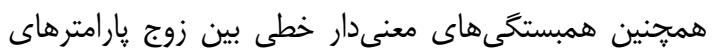

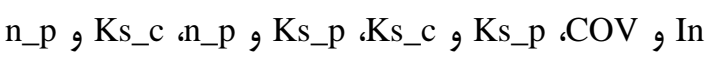
مشاهده مىشود. روابط غيرخطى نيز بين برخى از جار جفت

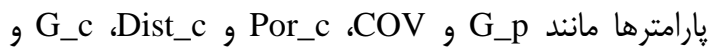
مشهود است. n_c با مقايسه نتايج اين تحقيق با نتايج مطالعه كمالى و

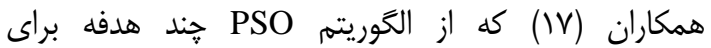

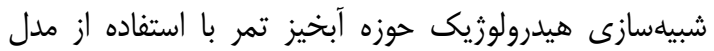

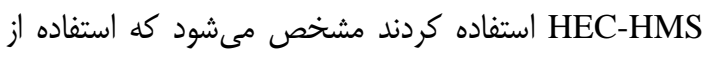

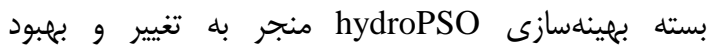

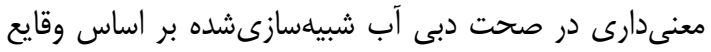

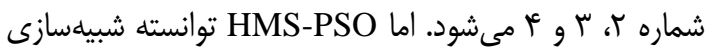

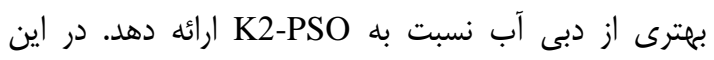

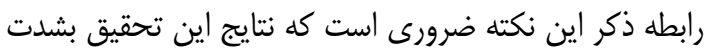

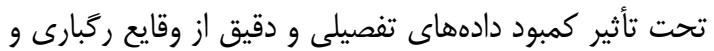

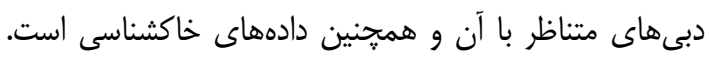

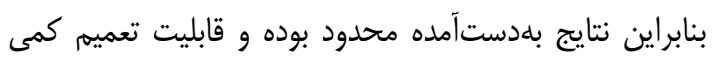

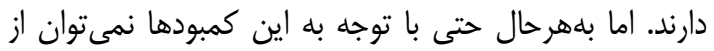

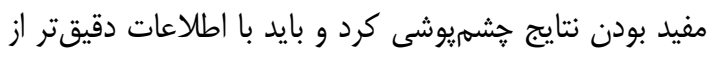

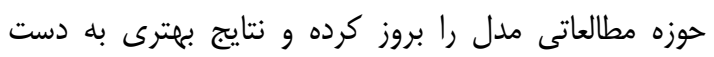

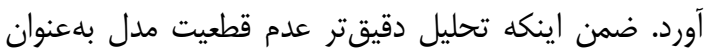
يك گزينه ييشنهادى براى مطالعات آينده مطرح مى فياشد.
در شكل (V) تكامل بهينه عمومى (بهترين اجراى مدل در

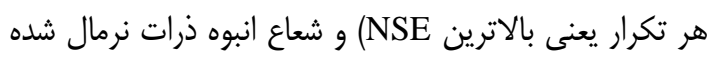

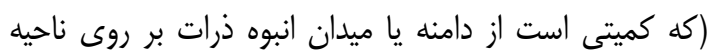

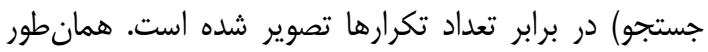

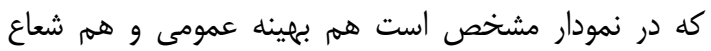

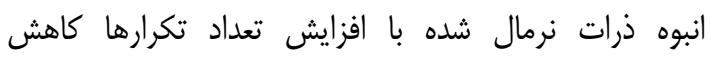

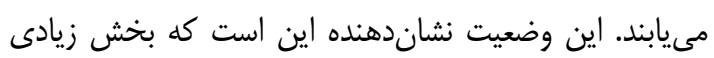

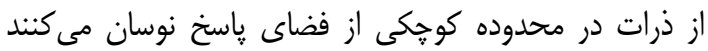

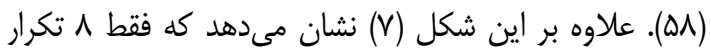

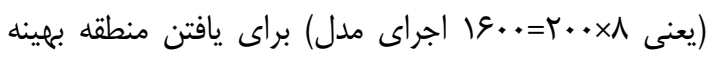
عمومى كافى است و ساير تكرارها فقط براى برائ بعبود و تصحيح جزئى جستجو بكار مىروند.

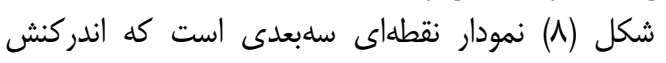

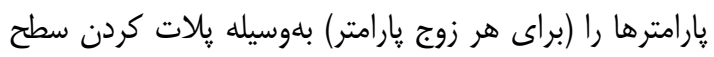

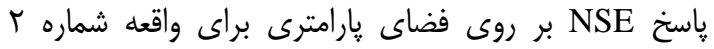

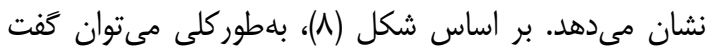

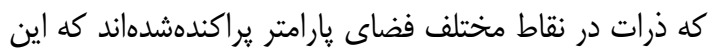

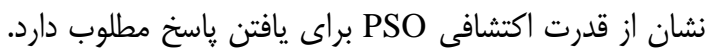

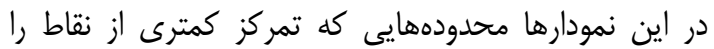

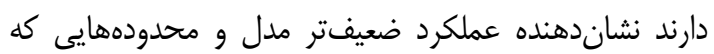

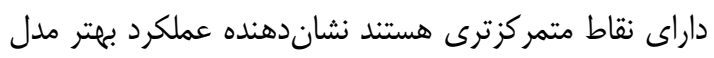

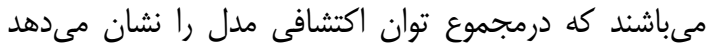

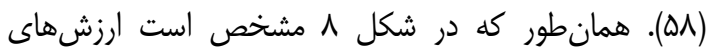

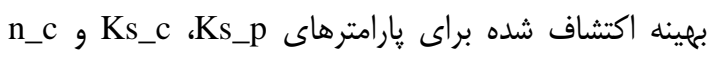

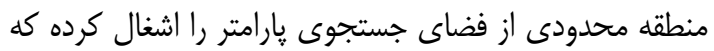

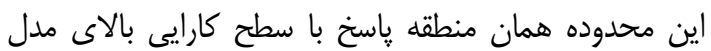

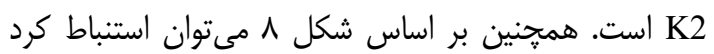

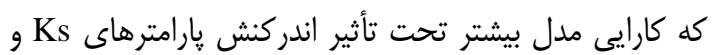

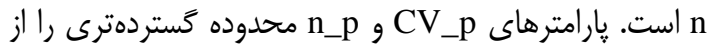

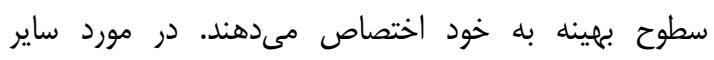

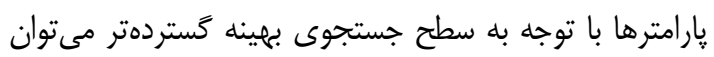

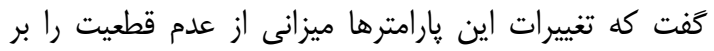

نتايج مدل تحميل خواهد كرد (FV).

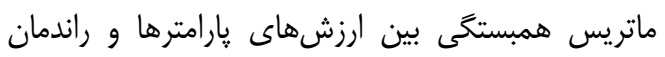

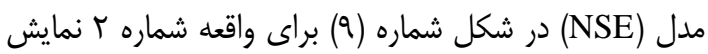



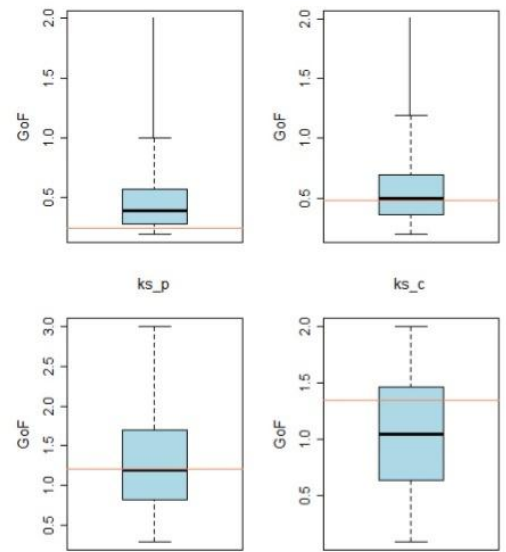

G_c

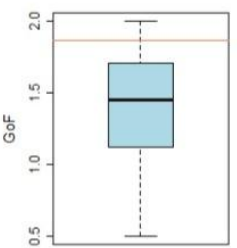

Dist_p

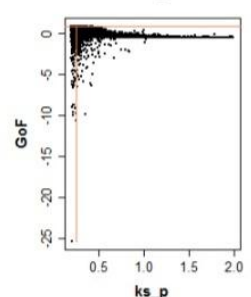

ks_p
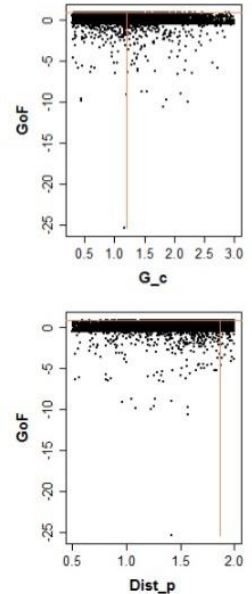

ks_c
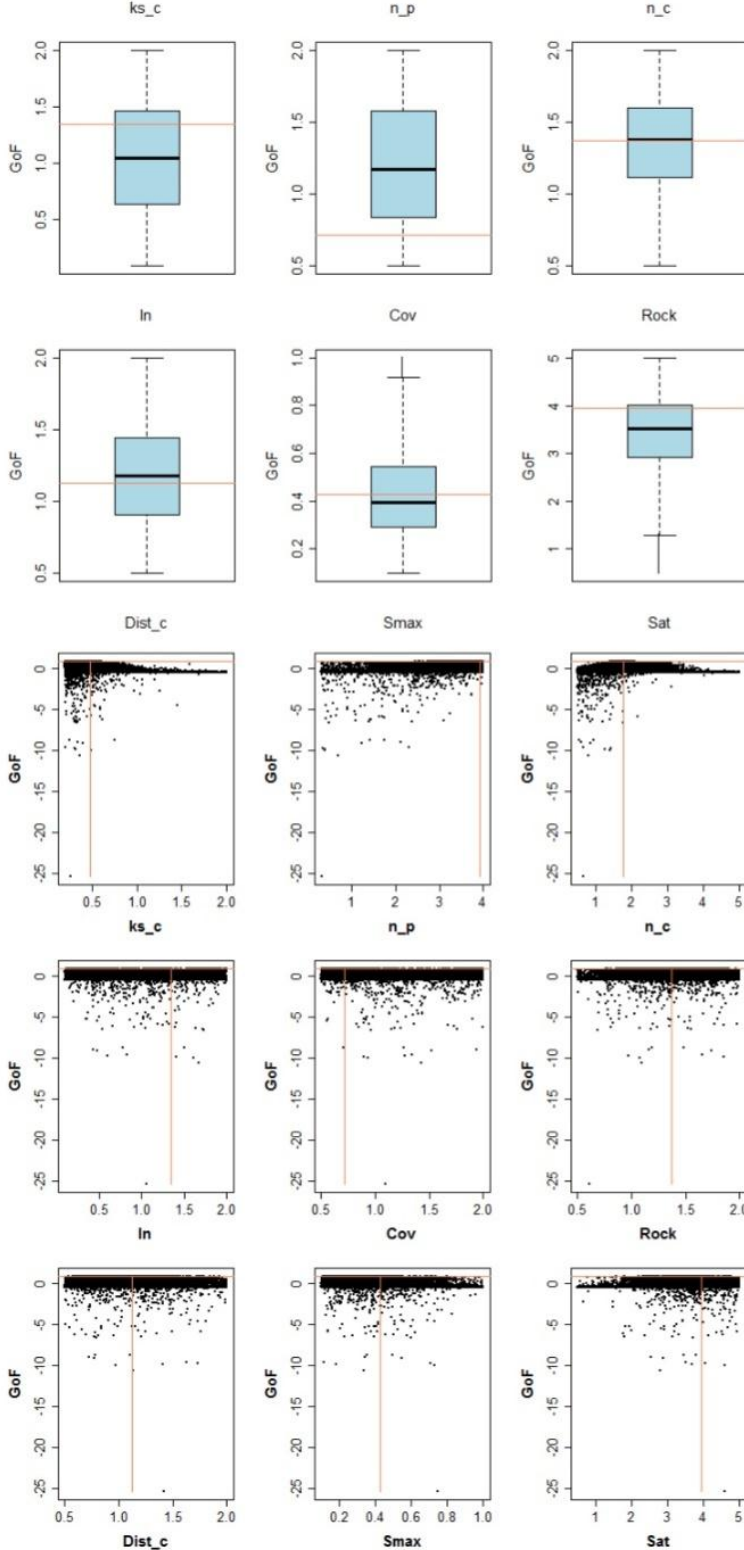

Rock

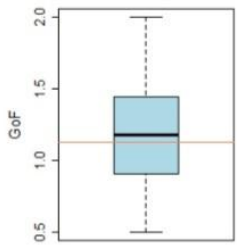

Dist_c
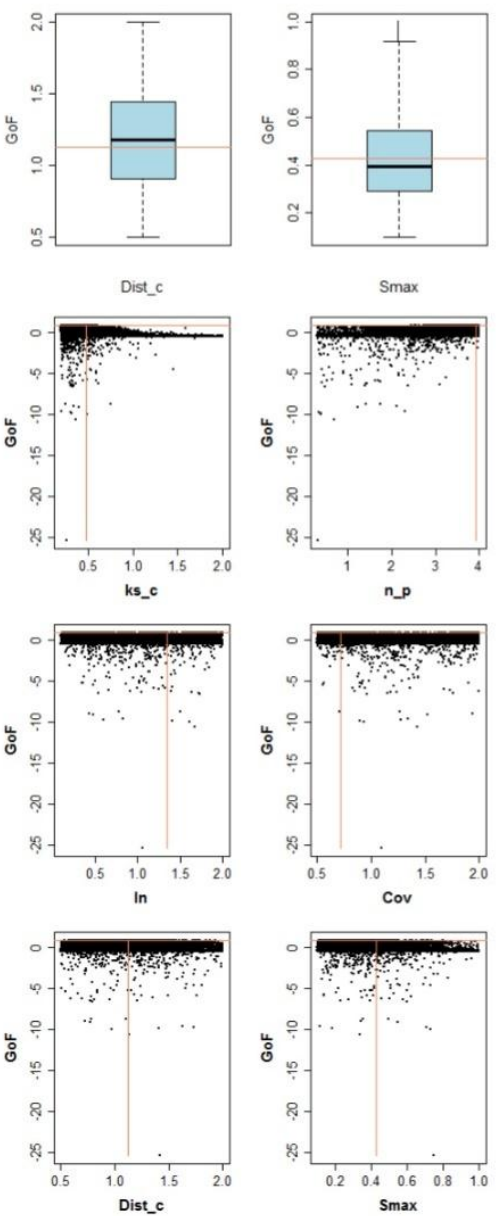

Smax

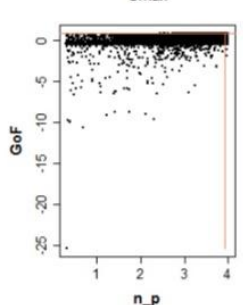

n_p
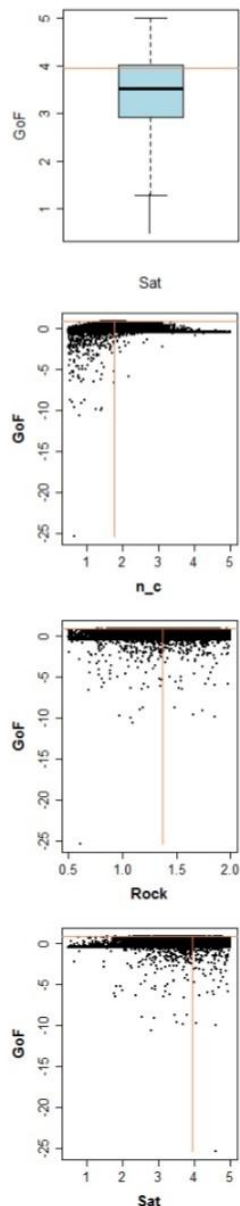

Sat

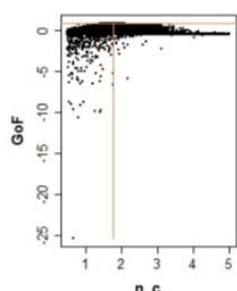

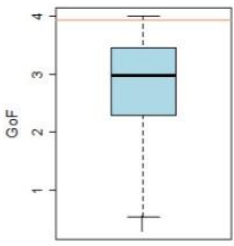
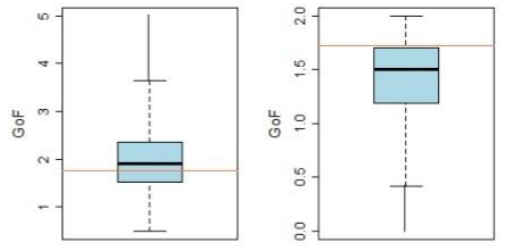

CV_p

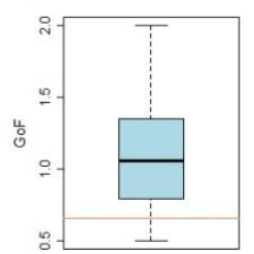

Por $\_$p
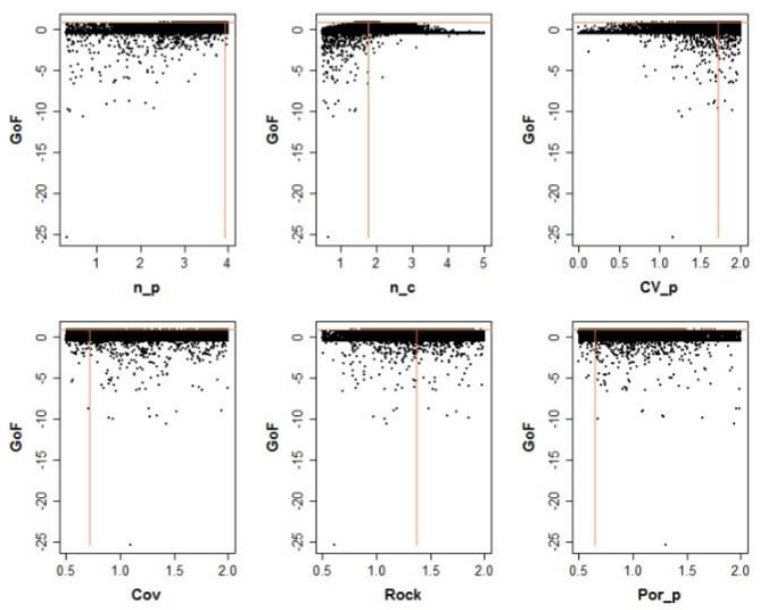

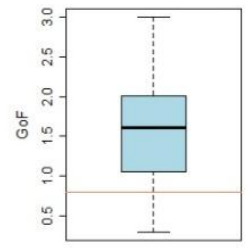

G_p

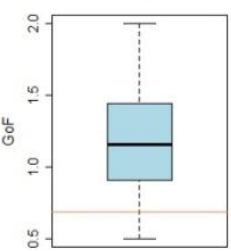

Por $-\mathrm{c}$

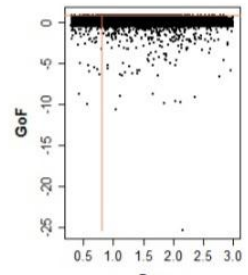

G_p

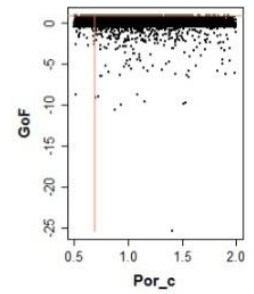

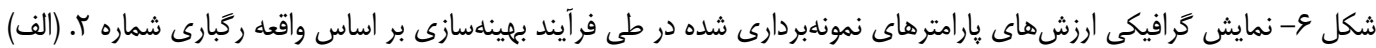

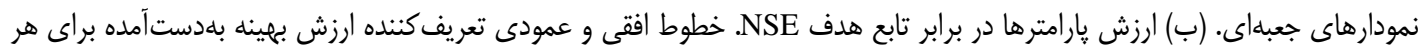
يارامتر است.

Figure 6. Graphical representation of parameters' values sampled during the optimization based on the event \#2. (a) Box-and-whisker plots (or boxplots). (b) Parameter values versus its equivalent NSE. Horizontal and vertical red line specifies the optimal value obtained for each parameter. 


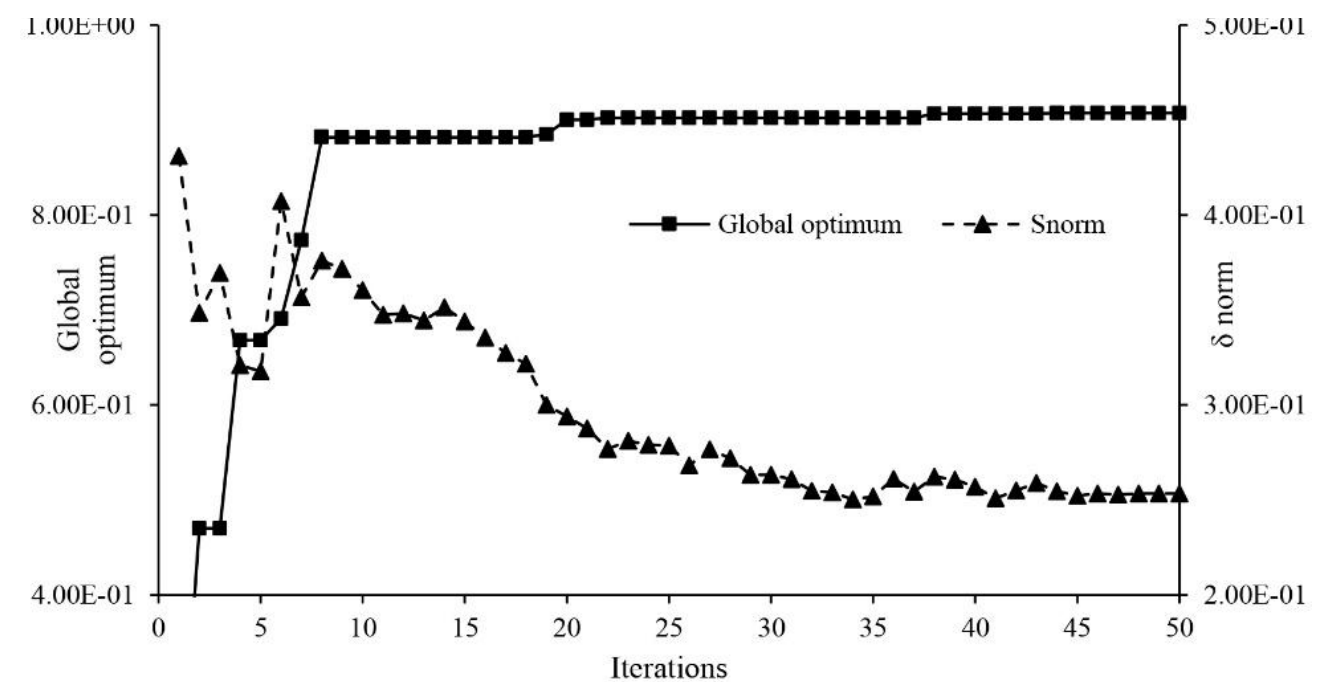

شكل V- تكامل بجينه عمومى (Global optimum) و شعاع انبوه ذرات نرمال شده (סnorm) در طى •ه تكرار مدل بر اساس واقعه

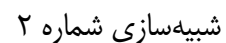

Figure 7. Evolution of the Global Optimum and the Normalized Swarm Radius ( $\delta$ norm) over the 50 model iterations
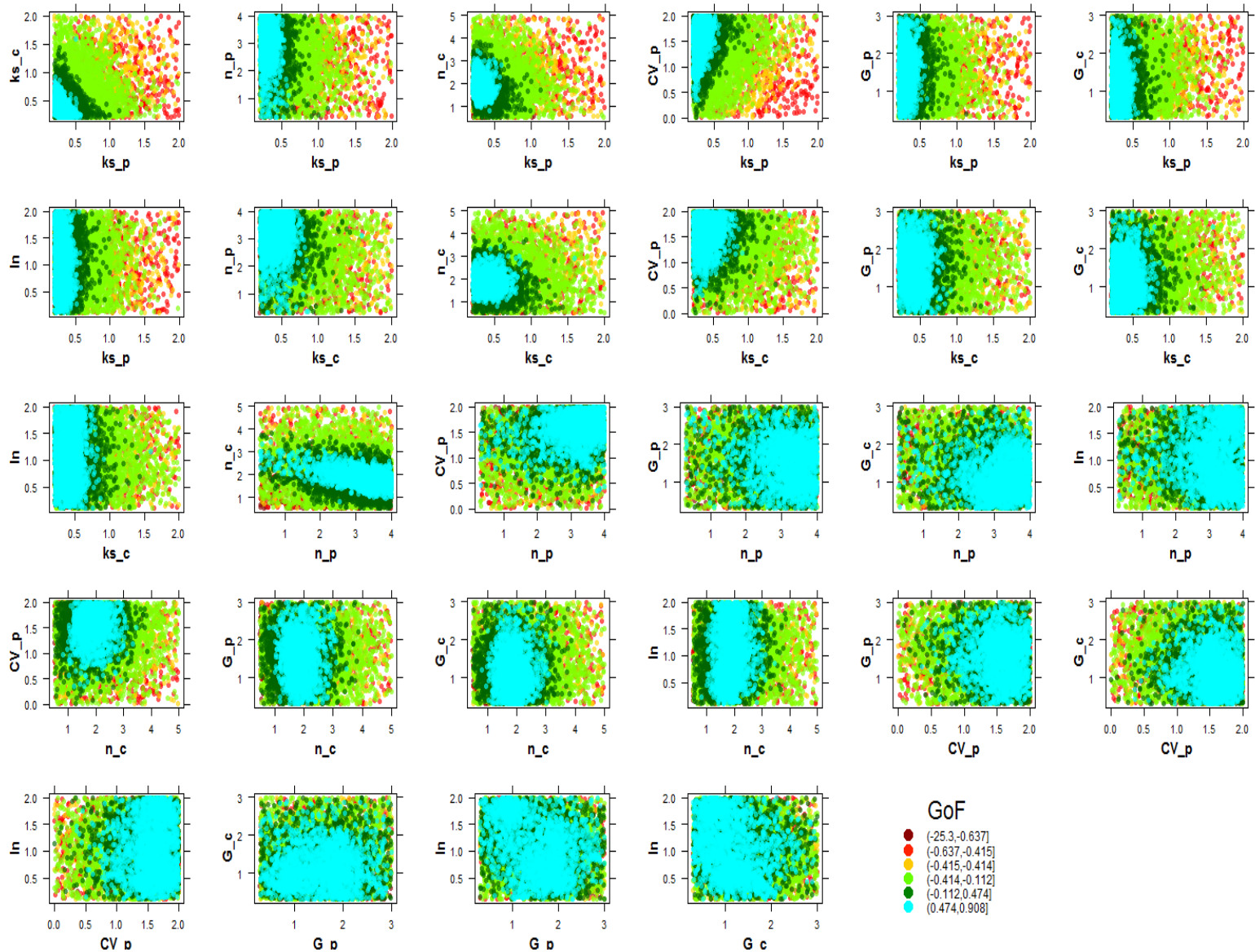

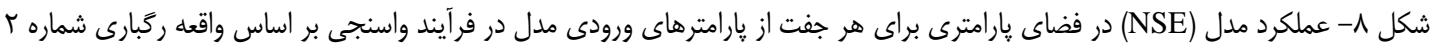

Figure 8. Model performance (NSE) projected onto the parameter space for different pairs of parameters based on the event \#2 


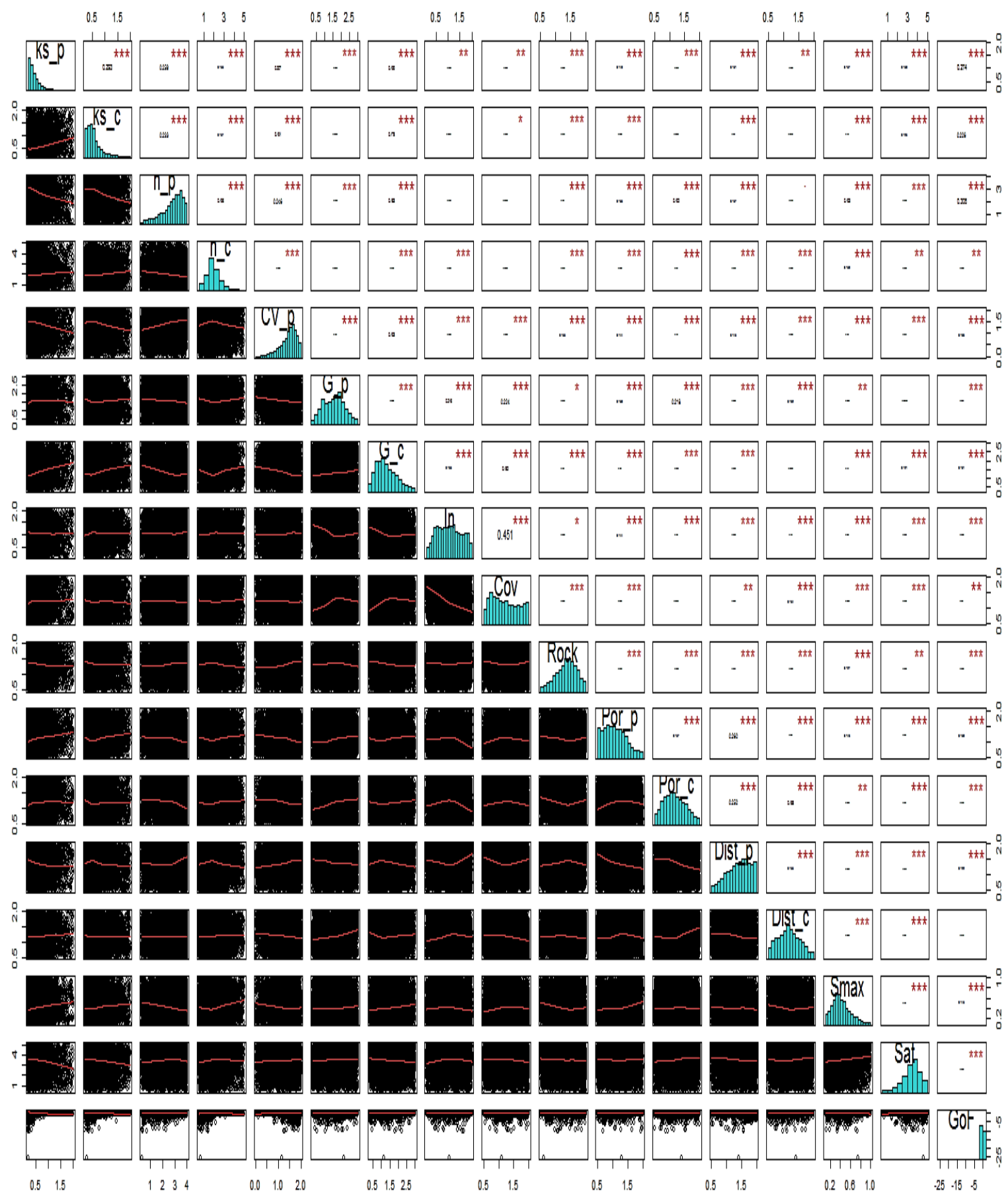

شكل q- ماتريس همبستخى بين پارامترها و تابع هدف NSE بر اساس شبيهسازى مبتنى بر واقعه شماره r

Figure 9. Correlation matrix between parameters and model performance (NSE), based on the event \#2. 
سيستماتيك دادههاى وردى است و دومى خطاى ناشى از

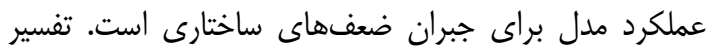

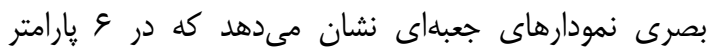
ين و Smax و ارزش بهينه يارامتر بهدستآمده در فرآيند واسنجى منطبق بر بر ميانه

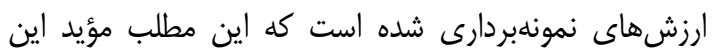

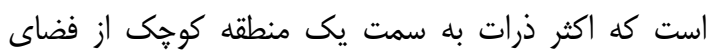

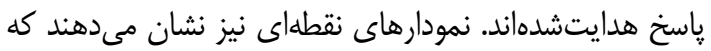

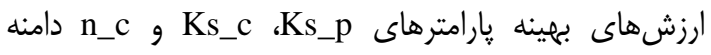

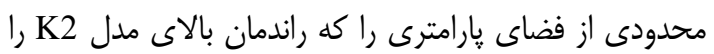

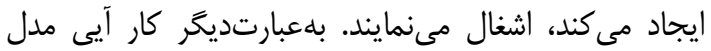

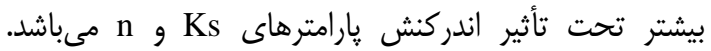

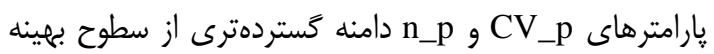

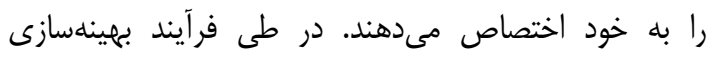

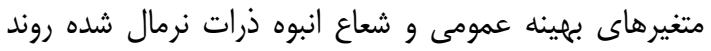

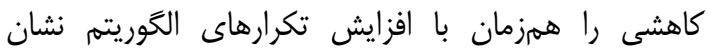

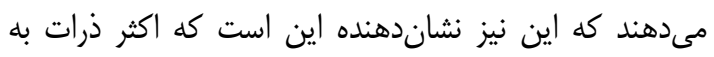

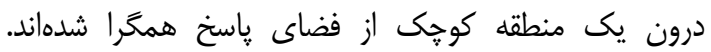

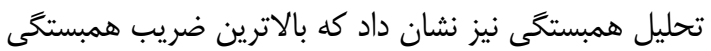

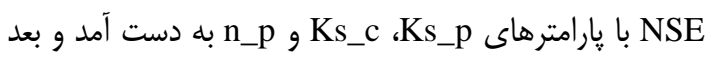

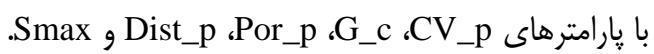

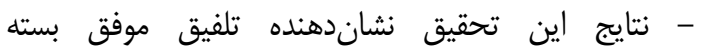

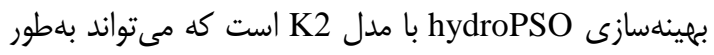

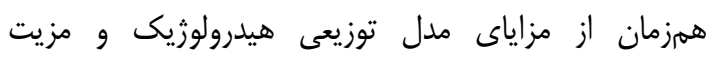
محاسبات نرم و يردازش موازى بسته R بهره بحيرد.

\section{تشكر و قدردانى}

ثزوهش حاضر با حمايت مالى دانشكاه بيرجند تحت

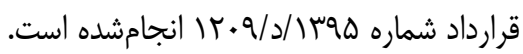

در اين مقاله بسته بهينهسازى hydroPSO بلمنظور واسنجى مدل بارش-رواناب KINEROS2 بر اساس براس 19

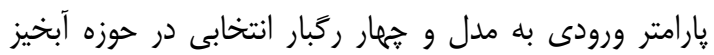
تمر در استان كلستان مورداستفاده قرار كرفت و ونتائ دنايج كليدى زير به دست آمد:

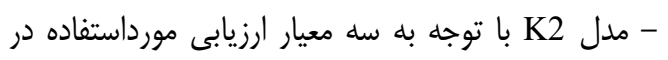

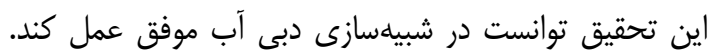

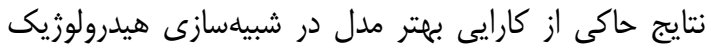

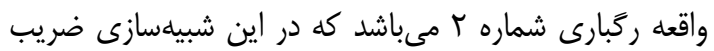

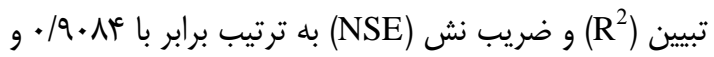

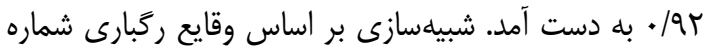

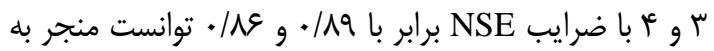

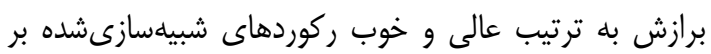

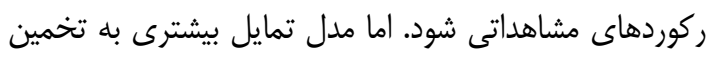

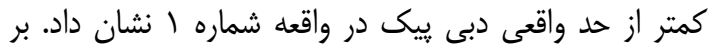

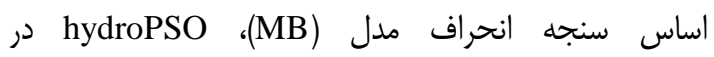

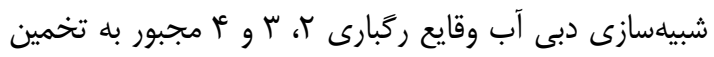

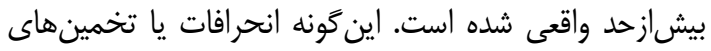

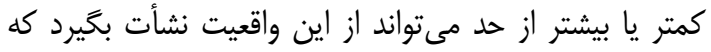

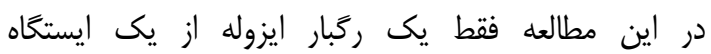
بارانسنجى بر كل حوزه تعميميافته است.

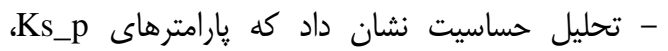

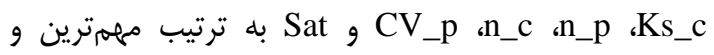

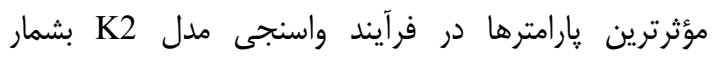

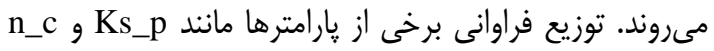

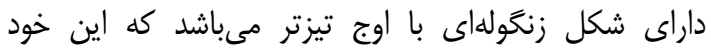

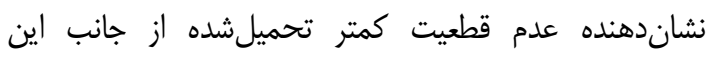

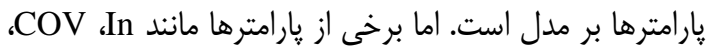
توزيع فراوانى يكنواختى را نشان مىدهند.

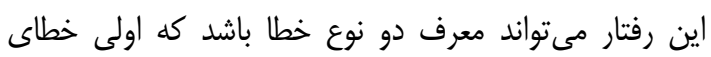


1. Abdelaziz, R. and M. Zambrano-Bigiarini. 2014. Particle swarm optimization for inverse modeling of solute transport in fractured gneiss aquifer. Journal of contaminant hydrology, 164: 285-298.

2. Al-Qurashi, A., N. McIntyre, H. Wheater and C. Unkrich. 2008. Application of the Kineros2 rainfall-runoff model to an arid catchment in Oman. Journal of Hydrology, 355(1): 91-105.

3. Azizian, A. and A.Shokoohi. 2014. DEM resolution and stream delineation threshold effects on the results of geomorphologic-based rainfall runoff models. Turkish Journal of Engineering and Environmental Sciences, 38(1): 64-78.

4. Baltar, A. and D.G. Fontane. 2004. A multiobjective particle swarm optimization model for reservoir operations and planning. Department of Civil and Environmental Engineering, Colorado State University, Fort Collins, Colo. $10 \mathrm{pp}$.

5. Beven, K. and A. Binley.1992. The future of distributed models: model calibration and uncertainty prediction. Hydrological processes, 6(3): 279-298.

6. Bloomfield, V.A. 2014. Using R for Numerical Analysis in Science and Engineering. CRC Press, $359 \mathrm{pp}$.

7. Canfield, H. and D. Goodrich. 2006. Differentiating the impact of parameter lumping from the impact of geometric simplification in modeling runoff and erosion 1584. Hydrological Processes, 20(1): 17-35.

8. Cleveland, W.S. 1979. Robust locally weighted regression and smoothing scatterplots. Journal of the American statistical association, 74(368): 829-836.

9. Doherty, J., L. Brebber and P. Whyte. 1994. PEST: Model-independent parameter estimation. Watermark Computing, Corinda, Australia, $122 \mathrm{pp}$.

10.Gholami, V. and M. Mohseni Saravi. 2010. Effects of impervious surfaces and urban development on runoff generation and flood hazard in the Hajighoshan watershed. Caspian Journal of Environmental Sciences, 8(1): 1-12.

11.Gill, M.K., Y.H. Kaheil, A. Khalil, M. McKee and L. Bastidas. 2006. Multiobjective particle swarm optimization for parameter estimation in hydrology. Water Resources Research, 42(7).

12.Guber, A.K., A.M. Yakirevich, AM. Sadeghi, Y.A. Pachepsky and D.R. Shelton. 2009. Uncertainty evaluation of coliform bacteria removal from vegetated filter strip under overland flow condition. Journal of environmental quality, 38(4): 1636-1644.

13. Guber, A.K., Y.A. Pachepsky, A.M. Yakirevich, D.R. Shelton, A.M. Sadeghi, D.C. Goodrich and C.L. Unkrich. 2011. Uncertainty in modelling of faecal coliform overland transport associated with manure application in Maryland. Hydrological processes, 25(15): 2393-2404.

14.Gupta, H.V., S. Sorooshian and P.O. Yapo. 1999. Status of automatic calibration for hydrologic models: Comparison with multilevel expert calibration. Journal of Hydrologic Engineering, 4(2): 135-143.

15.Hernandez, M., S.N. Miller, D.C. Goodrich, B.F. Goff, W.G. Kepner, C.M. Edmonds and K.B. Jones. 2000. Modeling runoff response to land cover and rainfall spatial variability in semi-arid watersheds. In Monitoring Ecological Condition in the Western United States (pp: 285-298). Springer Netherlands.

16.Jiang, Y., C. Liu, C. Huang and X. Wu. 2010. Improved particle swarm algorithm for hydrological parameter optimization. Applied Mathematics and Computation, 217(7): 32073215.

17.Kamali, B., S.J. Mousavi and K.C. Abbaspour. 2013. Automatic calibration of HEC-HMS using single-objective and multi-objective PSO algorithms. Hydrological Processes, 27(26): 40284042 .

18. Kalin, L. and M.M. Hantush. 2003. Evaluation of sediment transport models and comparative application of two watershed models. US Environmental Protection Agency, Office of Research and Development, National Risk Management Research Laboratory, $81 \mathrm{pp}$.

19. Kasmaei, L.P., R. Van Der Sant, P.J. Lane and G. Sheriadan. 2015. Modelling overland flow on burned hillslopes using the KINEROS2 model. $21^{\text {st }}$ International Congress on Modelling and Simulation, Gold Coast, Australia, 29 Nov t0 4 Dec 2015. 222-228.

20.Kennedy, J. and R. Eberhart. 1995. Particle swarm optimization. In: Proceedings IEEE International Conference on Neural Networks, pp: 1942-1948.

21.Kennedy, J.R., D.C. Goodrich and C.L. Unkrich. 2012. Using the KINEROS2 modeling framework to evaluate the increase in storm runoff from residential development in a semiarid environment. Journal of Hydrologic Engineering, 18(6): 698-706. 
22. Koster, G. 2013. Mapping runoff and erosion to reduce urban flooding and sediment flow towards sea, A case study on the Playa catchment, Bonaire. MSc. Thesis. Water Resources Management Group, WAGENINGEN University, $81 \mathrm{pp}$.

23. Martínez-Carreras, N., M. Soler, E. Hernández and F. Gallart. 2007. Simulating badland erosion with KINEROS2 in a small Mediterranean mountain basin (Vallcebre, Eastern Pyrenees). Catena, 71(1): 145-154.

24.McCuen, R.H. 1989. Hydrologic analysis and design (pp: 143-147). Englewood Cliffs, NJ: Prentice-Hall.

25.McCuen, R.H. and W.M. Snyder. 1975. A proposed index for comparing hydrographs. Water Resources Research, 11(6): 1021-1024.

26. Memarian, H., S.K. Balasundram, J. Talib, C.B.S. Teh, M.S. Alias, K.C. Abbaspour and A. Haghizadeh. 2012. Hydrologic Analysis of a Tropical Watershed using KINEROS2. Environment Asia, 5(1): 84-93

27. Memarian, H., S.K. Balasundram, J.B. Talib, C. Teh Boon Sung, A. Mohd Sood and K.C. Abbaspour. 2013. KINEROS2 application for land use/cover change impact analysis at the Hulu Langat Basin, Malaysia. Water and Environment Journal, 27(4): 549-560.

28. Meyer, P.D., M.L. Rockhold and G.W. Gee. 1997. Uncertainty analyses of infiltration and subsurface flow and transport for SDMP sites (No. NUREG/CR--6565; PNNL--11705). Nuclear Regulatory Commission, Washington, DC (United States). Div. of Regulatory Applications; Pacific Northwest National Lab., Richland, WA (United States).

29. Michaud, J.D. and S. Sorooshian. 1994. Effect of rainfall-sampling errors on simulations of desert flash floods. Water Resources Research, 30(10): 2765-2775.

30. Mirzaei, M., Y.F. Huang, A. El-Shafie, T. Chimeh, J. Lee, N. Vaizadeh and J. Adamowski. 2015. Uncertainty analysis for extreme flood events in a semi-arid region. Natural Hazards, 78(3): 1947-1960.

31. Molaeifar, A. 2013. KINEROS2 evaluation for hydrograph simulation of Ziarat watershed. M.Sc. Thesis, Gorgan University of Agriculture and Natural Resources, 95 pp (In Persian).

32.Moriasi, D.N., J.G. Arnold, M.W. Van Liew, R.L. Bingner, R.D. Harmel and T.L. Veith. 2007. Model evaluation guidelines for systematic quantification of accuracy in watershed simulations. Transactions of the ASABE, 50(3): 885-900.

33. Musau, J., J. Sang, J. Gathenya, E. Luedeling and P. Home. 2015. SWAT model parameter calibration and uncertainty analysis using the HydroPSO R package in Nzoia Basin, Kenya. Journal of Sustainable Research in Engineering, 1(3): 17-29.

34. Nachtergaele, F., H. Van Velthuizen, L. Verelst, N. Batjes, K. Dijkshoorn, V. Van Engelen and S. Prieler. 2008. Harmonized world soil database. Food and Agriculture Organization of the United Nations.

35. Namavar, B. 2011. Runoff simulation in Kameh watershed using Kineros2 and AGWA-GIS. M.Sc. thesis, Ferdowsi University of Mashhad, 85 pp (In Persian).

36. Nash, J.E. and J.V. Sutcliffe. 1970. River flow forecasting through conceptual models part I-A discussion of principles. Journal of hydrology, 10(3): 282-290.

37.Nearing, M.A., V. Jetten, C. Baffaut, O. Cerdan, A. Couturier, M. Hernandez and V. Souchère. 2005. Modeling response of soil erosion and runoff to changes in precipitation and cover. Catena, 61(2): 131-154.

38.Neitsch, S.L., J.R. Williams, J.G. Arnold and J.R. Kiniry. 2011. Soil and water assessment tool theoretical documentation version 2009. Texas Water Resources Institute.

39. Nikolova, M., S. Nedkov, V. Nikolov, I. Zuzdrov, M. Genev, T. Kotsev and Y. Krumova. 2009. Implementation of the" KINEROS" model for estimation of the flood prone territories in the Malki Iskar River basin. Information and Security, 24, 76.

40. Onstad, C.A. and D.L. Brakensiek. 1968. Watershed simulation by stream path analogy. Water Resources Research, 4(5): 965-971.

41.Parsopoulos, K.E. and M.N. Vrahatis. 2002. Recent approaches to global optimization problems through particle swarm optimization. Natural computing, 1(2-3): 235-306.

42.Poli, R., J. Kennedy and T. Blackwell. 2007. Particle swarm optimization. Swarm intelligence, 1(1): 33-57.Rovey, E.W. 1977. A distributed kinematic model of upland watersheds. Hydrology papers (Colorado State University); no. 93.

43. Safari, A., F. De Smedt and F. Moreda. 2012. WetSpa model application in the distributed model intercomparison project (DMIP2). Journal of Hydrology, 418: 78-89.

44.Schaffner, M., C.L. Unkrich and D.C. Goodrich. 2010. Application of the KINEROS2 site specific model to south-central NY and northeast PA: forecasting gaged and ungaged fast responding watersheds. NWS Eastern Region Technical Attatchment, 1, 2010. 
45.Semmens, D.J., D.C. Goodrich, C.L. Unkrich, R.E. Smith, D.A. Woolhiser and S.N. Miller. 2008. KINEROS2 and the AGWA modelling framework. In Hydrological modelling in arid and semi-arid areas, Wheater, H., Sorooshian, S. and Sharma, K.D. (eds.). Cambridge University Press, New York. 49-69.

46. Shafiei, M., B. Ghahraman, B. Saghafian, K. Davary, S. Pande and M. Vazifedoust. 2014. Uncertainty assessment of the agro-hydrological SWAP model application at field scale: A case study in a dry region. Agricultural Water Management, 146: 324-334.

47. Shen, Z.Y., L. Chen and T. Chen. 2012. Analysis of parameter uncertainty in hydrological and sediment modeling using GLUE method: a case study of SWAT model applied to Three Gorges Reservoir Region, China. Hydrology and Earth System Sciences, 16(1): 121-132.

48. Smith, R.E., D.C. Goodrich and C.L. Unkrich.1999. Simulation of selected events on the Catsop catchment by KINEROS2: a report for the GCTE conference on catchment scale erosion models. Catena, 37(3): 457-475.

49. Smith, R.E. and J.Y. Parlange.1978. A parameter-efficient hydrologic infiltration model. Water Resources Research, 14(3): 533-538.

50. Sorooshian, S. and V.K. Gupta. 1983. Automatic calibration of conceptual rainfall-runoff models: The question of parameter observability and uniqueness. Water Resources Research, 19(1): 260-268.

51.Sorooshian, S. and V.K. Gupta. 1995. Model calibration. Computer models of watershed hydrology, 23-68.

52. Van Griensven, A., T. Meixner, S. Grunwald, T. Bishop, M. Diluzio and R. Srinivasan. 2006. A global sensitivity analysis tool for the parameters of multi-variable catchment models. Journal of hydrology, 324(1): 10-23.

53. Vatseva, R., S. Nedkov, M. Nikolova and T. Kotsev. 2008. Modeling land cover changes for flood hazard assessment using Remote Sensing data. In Geospatial crossroads@ GI Forum’08Proceedings of the Geoinformatics Forum Salzburg, pp: 262-267.

54. Vrugt, J.A. and B.A. Robinson. 2007. Improved evolutionary optimization from genetically adaptive multimethod search. Proceedings of the National Academy of Sciences, 104(3): 708711 .

55.Wagener, T. and S.W. Franks. 2005. Regional Hydrological Impacts of Climatic Change: Hydroclimatic variability (Vol. 2). International Assn of Hydrological Sciences.

56. Woolhiser, D.A., C.L. Hanson and A.R. Kuhlman. 1970. Overland flow on rangeland watersheds. Journal of Hydrology (NZ), 9(2): 336-356.

57. Woolhiser, D.A., R.E. Smith and D.C. Goodrich. 1990. KINEROS: a kinematic runoff and erosion model: documentation and user manual. US Department of Agriculture, Agricultural Research Service, $139 \mathrm{pp}$.

58.Zambrano-Bigiarini, M. and R. Rojas. 2013. A model-independent Particle Swarm Optimisation software for model calibration. Environmental Modelling and Software, 43: 5-25. 


\title{
Parameters Optimization of KINEROS2 using Particle Swarm Optimization Algorithm for Single Event Rainfall-Runoff Simulation (Case Study: Tamar Watershed, Golestan, Iran)
}

\author{
Hadi Memarian ${ }^{1}$, Mohsen Pourreza Bilondi ${ }^{2}$ and Zinat Komeh ${ }^{3}$
}

1- Assistant Professor, Faculty of Natural Resources and Environment, Department of Watershed Management, University of Birjand, Birjand, Iran (Corresponding author: hadi_memarian@birjand.ac.ir)

2- Assistant Professor, Faculty of Agriculture, Department of Water Engineering, University of Birjand, Birjand, Iran

3- GIS Senior Expert, Faculty of Natural Resources and Environment, University of Birjand, Birjand, Iran Received: January 12, 2017 Accepted: July 3, 2017

\begin{abstract}
Simulation of rainfall-runoff process for planning and management of water resources and watersheds requires the use of a conceptual optimized hydrological model. In this study, the hydroPSO package was employed to optimize KINEROS2 (K2) parameters applied in the Tamar watershed, Iran. Four storm events were utilized in hydrograph simulation. Results indicated better efficiency of $\mathrm{K} 2$ based on the event \#2 with the coefficient of determination and Nash-Sutcliffe Efficiency (NSE) of 0.9084 and 0.92, respectively. The events \#3 and \#4 with NSE of 0.89 and 0.86 showed the excellent and very good fitness of simulated flow compared to observed flow, respectively. Sensitivity analysis established that the parameters Ks_p, Ks_c, $\mathrm{n} \_\mathrm{p}, \mathrm{n} \_\mathrm{c}, \mathrm{CV} \_\mathrm{p}$ and Sat were the most effective parameters in $\mathrm{K} 2$ calibration, respectively. The posterior distributions of some parameters such as Ks_p and n_c appeared to be more sharply peaked than other parameters which established less uncertainty in hydrological modeling. Visual inspection of boxplots showed that for 6 out of 16 parameters (Ks_c, n_c, G_c, Rock, Dist_c and Smax) the optimum value found during the optimization coincided with the median of all the sampled values confirming that most of the particles converged into a small region of the solution space. Dotty plots showed that the optimum values found for Ks_p, Ks_c, and n_c define a narrow range of the parameter space with high model performance. On the other hand, the model performance was more impacted by the interaction of $\mathrm{Ks}$ and $\mathrm{n}$ parameters. The parameters CV_p and n_p showed a wider range of the optimized levels. Correlation analysis revealed that the highest linear correlation between the NSE and K2 parameters was obtained for the Ks_p, Ks_c and n_p, followed by CV_p, G_c, Por_p, Dist_p and Smax. Conclusively, this work demonstrated the capability of hydroPSO in optimization of the K2 as an event-based hydrological model.
\end{abstract}

Keywords: HydroPSO, KINEROS2, Particle Swarm Optimization (PSO), Rainfall-runoff, Simulation 